\title{
Multipole Coulomb interactions with several electrons per crystal site: Crystal and mean fields, symmetry lowering, and loss of magnetic moments
}

\author{
A. V. Nikolaev* \\ Department of Physics, University of Antwerp, Campus Drie Eiken, Universiteitsplein 1, Antwerpen, Belgium
}

(Received 13 June 2004; revised manuscript received 24 September 2004; published 4 April 2005)

\begin{abstract}
A review of the technique of multipole expansion of the Coulomb interaction for a few electrons is presented. The correlation effects described by this multireference approach are beyond the methods based on a single determinantal wave function (standard electron band structure calculation and molecular orbital method). Starting with the Coulomb law, we use the technique of multipole expansion to calculate the crystal electric field, mean field, and symmetry lowering for a number of many-electron configurations $\left(f^{3}, s f^{3}, p f^{3}\right.$, and $d f^{3}$ ). We consider these configurations as very relevant for a model where three $f$ electrons are localized and the fourth electron is delocalized and can be expanded locally in $s^{-}, p^{-}, d$-states in the spirit of the tight binding model. Prompted by the ideas of the double exchange, we study the intrasite multipole interaction, which couples localized electrons $\left(f^{3}\right)$ to delocalized ones on the same crystal site. We show that this interaction may be responsible for an effective loss of magnetic moments when a suitable symmetry lowering takes place. The present approach can be considered as a microscopic foundation of Kondo demagnetization when the loss of magnetic moments occurs together with a structural phase transition. The approach may be relevant for cerium and $\mathrm{NpO}_{2}$.
\end{abstract}

DOI: 10.1103/PhysRevB.71.165102

PACS number(s): 71.10.Li, 75.10.Dg

\section{INTRODUCTION}

Recently, a new technique of multipole expansion of the Coulomb interaction was presented in a number of publications. ${ }^{1-3}$ First it was applied to a model of cerium with only one $4 f$ electron, which feels the Coulomb repulsion from neighboring electrons. ${ }^{1}$ Later, it was generalized for the case of a $4 f 5 d$ model where the $4 f$ electron is strongly coupled with a delocalized $5 d$ electron. ${ }^{2}$ The main reason for introducing such models is that, despite progress in the field of electron band structure calculations, ${ }^{4}$ fundamental important intrasite correlations giving rise to atomic term splittings are routinely ignored. ${ }^{2}$ The intrasite correlations are typical for atoms with open electron shells. When an atom has two or more valence electrons on orbitally degenerate states (like $d$ - or $f$-), its energy spectrum can be very complex, reflecting the electronic degrees of freedom. ${ }^{5}$ There exist empirical observations known as Hund's rules, which prescribe the occupation of the orbitals, but those are just consequences of the atomic theory of many-electron states formulated by Condon and Shortley long ago. ${ }^{5}$ The real driving force behind the term splitting is the Coulomb repulsion of the valence electrons. In many atoms the splitting between the highest and the lowest electron levels is of an order of few electronvolts, which is of the same magnitude as the electron band effects. Therefore, the full consideration of intrasite interactions should be a necessary ingredient of a many-electron theory aiming to capture these missed correlations.

As was mentioned before, ${ }^{2}$ the failure of the standard band structure approach to describe such effects is rather serious. The band structure calculation assumes a solution with only one Slater determinant and one electronic configuration. From a quantum-chemical viewpoint this picture corresponds to a single determinant Hartree-Fock calculation scheme without any correlation effects. Correlations appear only if one takes into account several Slater determinants. Such a calculation scheme is called configuration interaction (CI) or, more precise, multireference approach.

The multipole Coulomb expansion, on the other hand, requires many Slater determinants ${ }^{2,3}$ (multireference treatment) and, from this point of view, it represents a genuine manyelectron method. It also provides a unified description of the crystal field effects and the atomic term splittings. The intrasite Coulomb repulsion, which is responsible for Hund's rules, is included on equal footing with the spin-orbit coupling, and the crystal field effects. For a single site the technique is equivalent to the classical description of atomic terms. ${ }^{5}$ Moreover, the model calculations of multiplet spectra of the $\mathrm{C}_{60}$ molecular ion based on the multipole expansion ${ }^{3}$ are $\operatorname{close}^{6}$ to a sophisticated and advanced complete active space self-consistent-field (CASSCF) method. ${ }^{7}$

In the present work we develop and accomplish the technique of multipole Coulomb interactions for the case of few electrons. Another motivation is to clarify the microscopic foundation of the intrasite coupling between localized and conduction electrons. Such an interaction is often referred to as the Anderson hybridization. ${ }^{8}$ However, the coupling was discussed already by Zener 10 years earlier. ${ }^{9}$ Zener considered the coupling between an incomplete $d$-shell and the conduction electrons, and concluded that, in accordance with Hund's rules, it tends to align the spins in a ferromagnetic manner. (This is the third principle of his double-exchange approach. ${ }^{9}$ ) Since the multipole Coulomb interactions offer a microscopic basis for Hund's rules and can even describe the exceptions to them for a number of elements ${ }^{5}$ (like atomic cerium with the ${ }^{1} G_{4}$ ground state ${ }^{10}$ ), we can apply the technique to derive the intrasite coupling from the Coulomb repulsion, i.e., from first principles. Depending on the interplay between the crystal symmetry and energy splittings, the out- 
come of this coupling may differ. As was shown by Zener, the coupling can lead to a ferromagnetic or antiferromagnetic ground state. ${ }^{9}$ In the following we will demonstrate that it also can be the cause for a nonmagnetic ground state (the so-called Kondo effect ${ }^{11}$ ). We consider this mechanism of demagnetization as a candidate for the $\gamma \rightarrow \alpha$ phase transition in cerium ${ }^{12}$ and for the symmetry lowering $F m \overline{3} m \rightarrow P n \overline{3} m$ in neptunium dioxide. ${ }^{13}$

The paper is organized as follows. In Sec. II we describe our method for treating local electron configurations. Next (Sec. III) we examine thoroughly the case of three localized $f$ electrons. In Sec. IV we generalize the $f^{3}$ model by including the multipole interactions with a valence electron which is present instantaneously on the same site. We show that, in the framework of this extended model, the disappearance of the magnetic moments can be understood and ascribed to the trigonal symmetry lowering. Our conclusions are summarized in Sec. V.

\section{MANY-ELECTRON CALCULATION}

In this section we describe in detail how we construct the basis consisting of many determinantal wave functions for a many-electron system and how we calculate the relevant matrix elements. As a model system we consider $\mathrm{NpO}_{2}$ with three localized $5 f$ electrons at the neptunium site, although the technique is general and can be applied to other cases. First, we perform a band structure calculation in order to determine the conduction electron charge distribution in the muffin-tin (MT) sphere around Np. Subsequently, we apply the configuration interaction method to treat the manyelectron system consisting of the localized $5 f^{3}$ electrons and the conduction electron in the MT sphere.

\section{A. Electron band structure calculation}

We have started by performing an electron band structure calculation of $\mathrm{NpO}_{2}$ using our linear augmented plane-wave (LAPW) code. ${ }^{14}$ The calculation has been done assuming the muffin-tin (MT) shape of the one-electron potential and the Barth-Hedin expression for exchange, ${ }^{15}$ which is a variant of the local density approximation (LDA). The equal MT radii $R_{M T}^{\mathrm{Np}}=2.2206$ and $R_{M T}^{O}=2.2206$ in atomic units (a.u.) were chosen for $\mathrm{Np}$ and $\mathrm{O}$, with the cubic lattice constant $a$ $=10.2567$ a.u. (or $5.4276 \AA$ ). ${ }^{27}$ The MT potential and density of $\mathrm{Np}$ and $\mathrm{O}$ have been obtained self-consistently using a LAPW basis of $\sim 300$ functions on a 20-point mesh of the irreducible part of the Brillouin zone. The three $5 f$ electrons of $\mathrm{Np}$ were treated as core states, which adjust selfconsistently to the conduction electron density.

As a result of the calculation we obtained that $\mathrm{NpO}_{2}$ is an insulator, with the energy gap $\Delta E=0.789 \mathrm{eV}$. The width of the occupied electron band below the Fermi level is $E_{T}$ $-E_{B}=5.953 \mathrm{eV}$. The spin-orbit splitting between $5 f_{7 / 2}$ and $5 f_{5 / 2}$ one-electron states is $\Delta_{f}=0.983 \mathrm{eV}$. The main goal of the calculation, however, was the electron charge density distribution inside the neptunium MT sphere. The calculated partial charges of different angular symmetry $(l=0-3)$ are quoted in Table I. An important result is that on average there
TABLE I. Angular-momentum-decomposed (partial) electronic charges $Q_{l}^{A}$ and total charges $Q^{A}$ inside neptunium and oxygen MT spheres and in the interstitial region (LAPW calculations; see Ref. 4 for details and definitions); $Q^{N p}=-\left(2 Q^{O}+Q^{i}\right)$.

\begin{tabular}{cccc}
\hline \hline$A$ & $\mathrm{~Np}$ & $\mathrm{O}$ & Interstices/unit \\
\hline$Q_{s}^{A}$ & $0.041 e$ & $0.027 e$ & -- \\
$Q_{p}^{A}$ & $0.401 e$ & $4.313 e$ & -- \\
$Q_{d}^{A}$ & $0.261 e$ & $0.046 e$ & -- \\
$Q_{f}^{A}$ & $2.185 e$ & $0.011 e$ & -- \\
$Q^{A}$ & $+4.108|e|$ & $-0.284|e|$ & $-3.540|e|$ \\
\hline \hline
\end{tabular}

is approximately one conduction electron present inside the Np MT sphere. Therefore, the localized $5 f^{3}$ configuration of $\mathrm{Np}$ cannot be considered separately from this valence electron, which can be of $7 s-, 7 p$-, or $6 d$-type. The instantaneous configuration at the neptunium site becomes $7 s 5 f^{3}, 7 p 5 f^{3}$, or $6 d 5 f^{3}$.

In all cases this additional electron experiences a strong Coulomb repulsion with the three localized partners. This interaction is not fully accounted for by the band structure calculations because it requires a multideterminant treatment or configuration interaction (CI). ${ }^{2}$ Therefore, we have to follow a different route; below, we study the electron spectrum using the multipole expansion of electronic densities.

\section{B. Many-electron basis states}

Our method of multipole expansion of the Coulomb interaction has been used before. ${ }^{1-3}$ Here, we formulate it in detail for the $s f^{3}$ configuration following Refs. 2 and 3. In Sec. III we deal with the $5 f^{3}$ configuration, which is easily obtained from $s f^{3}$ by omitting one $s$ electron. In Sec. IV we consider $7 s 5 f^{3}, 7 p 5 f^{3}$, and $6 d 5 f^{3}$ configurations. For the latter two cases we will describe the important differences with $s f^{3}$.

We start by considering a face-centered cubic (fcc) crystal of $N \mathrm{~Np}$ atoms. Each atomic site possesses one $7 s$ and three $5 f$ electrons. The position vector of an electron near a crystal lattice site $\vec{n}$ is given by

$$
\vec{R}(\vec{n})=\vec{X}(\vec{n})+\vec{r}(\vec{n}) .
$$

Here, $\vec{X}(\vec{n})$ is the lattice vector, which specifies the centers of the atoms (Np nuclei) on a rigid fcc lattice. The radius vector $\vec{r}(\vec{n})$ is given in polar coordinates by $[r(\vec{n}), \Omega(\vec{n})]$, where $r$ is the length and $\Omega=(\Theta, \phi)$ stands for the polar angles. We label the basis ket vectors at the lattice site $\vec{n}$ by a single index $I$ or, alternatively, by four one-electron indices $\left(i_{1}^{f}, i_{2}^{f}, i_{3}^{f} ; i^{s}\right)$

$$
|I\rangle_{\vec{n}}=\left|i_{1}^{f}, i_{2}^{f}, i_{3}^{f} ; i^{s}\right\rangle_{\vec{n}}
$$

The index $i^{f}=\left(m^{f}, s_{z}^{f}\right)$ stands for the orbital $\left(m^{f}=1-7\right)$ and spin projection $\left(s_{z}= \pm 1 / 2\right)$ quantum numbers of one $f$ electron. Therefore, there are 14 states which we label by $i^{f}$ $=1-14$. Two states of the $s$ electron are labeled by $i^{s}=1,2$. The many-electron basis wave functions are 


$$
\left\langle\vec{r}_{1}, \vec{r}_{2}, \vec{r}_{3}, \vec{r}_{4} \mid I\right\rangle_{\vec{n}}=\frac{1}{\sqrt{N_{a}}} \sum_{a} P(a) \prod_{t=1}^{3}\left\langle\vec{r}_{t} \mid i_{t}^{f}\right\rangle_{\vec{n}} \cdot\left\langle\vec{r}_{4} \mid i^{s}\right\rangle_{\vec{n}},
$$

where $a$ stands for a permutation of four electrons, the factor $P(a)= \pm 1$ takes into account the parity of the permutation $a$, $N_{a}$ is the number of the permutations, and

$$
\begin{aligned}
\left\langle\vec{r} \mid i^{f}\right\rangle_{\vec{n}} & =\mathcal{R}_{f}(r(\vec{n}))\left\langle\hat{n} \mid i^{f}\right\rangle, \\
\left\langle\vec{r}^{\prime} \mid i^{s}\right\rangle_{\vec{n}} & =\mathcal{R}_{s}\left(r^{\prime}(\vec{n})\right)\left\langle\hat{n}^{\prime} \mid i^{s}\right\rangle .
\end{aligned}
$$

Here, $\mathcal{R}_{f}$ and $\mathcal{R}_{s}$ are the radial components of the $5 f$ and $7 s$ electrons, respectively; $\hat{n}$ stands for $\Omega(\vec{n})$. The $5 f$ spinorbitals can be written as

$$
\left\langle\hat{n} \mid i^{f}\right\rangle=\left\langle\hat{n} \mid m_{f}\right\rangle u_{s}\left(s_{z}(f)\right) .
$$

Here, $u_{s}$ is the spin function $(s= \pm)$. The $f$-orbital parts, $\left\langle\hat{n} \mid m_{f}\right\rangle$, are expressed in terms of spherical harmonics $Y_{l}^{m}(\Omega)=\langle\hat{n} \mid l, m\rangle$. We find it convenient to work with real spherical harmonics ${ }^{16} Y_{l}^{\tau}$, where $\tau=0,(m, c)$ or $(m, s)$.

The order of indices in (2.2) is important. For example, as follows from the dynamical equivalence of the electrons, the state $\left|i_{1}^{f}, i_{2}^{f}, i^{s}, i_{3}^{f}\right\rangle$ can be reduced to $\left|i_{1}^{f}, i_{2}^{f}, i_{3}^{f} ; i^{s}\right\rangle$ by permuting the third and the fourth electrons, i.e.,

$$
\left|i_{1}^{f}, i_{2}^{f}, i^{s}, i_{3}^{f}\right\rangle=-\left|i_{1}^{f}, i_{2}^{f}, i_{3}^{f} ; i^{s}\right\rangle
$$

and so on. To describe the same quantum state we will use the basis vectors (2.2) and apply the permutation law (2.6) when needed. Alternatively, one can use the corresponding Slater determinants for the four-electron wave functions, Eq. (2.3). However, the permutation relations of the type of Eq. (2.6) are more efficient for our purposes. Excluding equivalent states, we find only $(14 \times 13 \times 12 / 3$ ! $) \times 2=728$ independent functions, or determinants, for $7 s 5 f^{3}$. (These are 364, 2184 , and 3640 for $5 f^{3}, 7 p 5 f^{3}, 6 d 5 f^{3}$, respectively.) Notice that every basis wave function is in fact a Slater determinant, Eq. (2.3).

\section{Multipole repulsion between electrons}

Now, we take into account the Coulomb intrasite and intersite repulsion by expanding the interaction in multipole series. As was discussed in Ref. 2, these interactions are treated exactly in the chosen quantum space $\left(7 s 5 f^{3}\right)$.

The Coulomb interaction between two electrons at sites $\vec{n}$ and $\vec{n}^{\prime}$ is given by

$$
V\left(\vec{R}(\vec{n}), \vec{R}^{\prime}\left(\vec{n}^{\prime}\right)\right)=\frac{1}{\left|\vec{R}(\vec{n})-\vec{R}^{\prime}\left(\vec{n}^{\prime}\right)\right|} .
$$

The multipole expansion in terms of site symmetry functions (SAFs) (Ref. 16) is

$$
V\left(\vec{R}(\vec{n}), \vec{R}^{\prime}\left(\vec{n}^{\prime}\right)\right)=\sum_{\Lambda \Lambda^{\prime}} v_{\Lambda \Lambda^{\prime}}\left(\vec{n}, \vec{n}^{\prime} ; r, r^{\prime}\right) S_{\Lambda}(\hat{n}) S_{\Lambda^{\prime}}\left(\hat{n}^{\prime}\right),
$$

where

$$
v_{\Lambda \Lambda^{\prime}}\left(\vec{n}, \vec{n}^{\prime} ; r, r^{\prime}\right)=\int d \Omega(\vec{n}) \int d \Omega^{\prime}\left(\vec{n}^{\prime}\right) \frac{S_{\Lambda}(\hat{n}) S_{\Lambda^{\prime}}\left(\hat{n}^{\prime}\right)}{\left|\vec{R}(\vec{n})-\vec{R}^{\prime}\left(\vec{n}^{\prime}\right)\right|} .
$$

The SAFs are linear combinations of spherical harmonics and transform as irreducible representations of a site point group; see Ref. 16. The index $\Lambda$ stands for $(l, \tau)$, with $\tau$ $=(\Gamma, \mu, k)$. Here, $l$ accounts for the angular dependence of the multipolar expansion, $\Gamma$ denotes an irreducible representation (in the present case the group is $O_{h}$ ), $\mu$ labels the representations that occur more than once, and $k$ denotes the rows of a given representation.

The intrasite case corresponds to $\vec{n}=\vec{n}^{\prime}$. The interaction function $v_{\Lambda \Lambda^{\prime}}\left(r, r^{\prime}\right) \equiv v_{\Lambda \Lambda^{\prime}}\left(\vec{n}=\vec{n}^{\prime} ; r, r^{\prime}\right)$ then becomes particularly simple

$$
v_{\Lambda \Lambda^{\prime}}\left(r, r^{\prime}\right)=\left(\frac{r_{<}^{l}}{r_{>}^{(l+1)}}\right) \frac{4 \pi}{2 l+1} \delta_{\Lambda \Lambda^{\prime}},
$$

where $r_{>}=\max \left(r, r^{\prime}\right), r_{<}=\min \left(r, r^{\prime}\right)$ and $\delta_{\Lambda \Lambda^{\prime}}=\delta_{\tau \tau^{\prime}} \delta_{l l^{\prime}}$. The last expression is also site independent.

There is no simple analytical expression for the intersite case, $\vec{n} \neq \vec{n}^{\prime} .{ }^{17}$ The intersite multipole interactions are anisotropic and, for practical purposes, it is important to use the following dependence: ${ }^{17}$

$$
v_{\Lambda \Lambda^{\prime}}\left(\vec{n}, \vec{n}^{\prime} ; r, r^{\prime}\right) \sim \frac{(r)^{l}\left(r^{\prime}\right)^{l^{\prime}}}{\left|\vec{X}(\vec{n})-\vec{X}\left(\vec{n}^{\prime}\right)\right|^{\mid l+l^{\prime}+1}} .
$$

\section{Intrasite matrix elements}

For the Coulomb interaction between four electrons on $a$ same site $\vec{n}$ we have a sum of six two-body terms

$$
V^{(4)}=\frac{1}{2} \sum_{t=1}^{4} \sum_{p(\neq t)=1}^{4} V\left(\vec{r}_{t}, \vec{r}_{p}\right)
$$

where each term is given by the multipole expansion (2.8). In order to calculate the matrix elements of $V^{(4)}$, $\left\langle i_{1}^{f}, i_{2}^{f}, i_{3}^{f} ; i^{s}\left|V^{(4)}\right| j_{1}^{f}, j_{2}^{f}, j_{3}^{f} ; j^{s}\right\rangle$, one has to classify the electronic transitions. Following Ref. 3, where the energy terms of molecular ions $\mathrm{C}_{60}^{m \pm}, m=2-5$, were calculated, we consider four possibilities for the fourth $s$ electron: (1) $i^{s} \rightarrow j^{s}$; (2) $i^{s}$ $\rightarrow j_{3}^{f}$; (3) $i^{s} \rightarrow j_{2}^{f}$; and (4) $i^{s} \rightarrow j_{1}^{f}$, which we label by the index $a_{4}=1-4$. The $a_{4}=2$ and $a_{4}=4$ transitions involve odd number of transpositions among $j_{1}^{f}, j_{2}^{f}, j_{3}^{f} ; j^{s}$, and the parity is $P\left(a_{4}=2\right)=P\left(a_{4}=4\right)=-1$. For two other transitions the number of transpositions is even and $P\left(a_{4}=1\right)=P\left(a_{4}=3\right)=1$. After this we are left with only three $j$ states, which we label as $j_{1}^{\prime}, j_{2}^{\prime}$, and $j_{3}^{\prime}$. For the next electron, $i_{3}^{f}$, we can consider three possibilities $\left(i_{3}^{f} \rightarrow j_{3}^{\prime}, i_{3}^{f} \rightarrow j_{2}^{\prime}, i_{3}^{f} \rightarrow j_{1}^{\prime}\right)$ which we label by the index $a_{3}=1-3$. In this way we continue until we exhaust all four electrons. As a result, each subcase (or electron transition) is classified by the three index label $a \equiv\left(a_{4}, a_{3}, a_{2}\right)$, and its parity is $P(a)=P\left(a_{4}\right) P\left(a_{3}\right) P\left(a_{2}\right)$. Mathematically, we reduce a permutation of four electrons to a product of transpositions. The matrix element $\left\langle I\left|V^{(4)}\right| J\right\rangle$ is found as ${ }^{18}$ 


$$
\left\langle I\left|V^{(4)}\right| J\right\rangle=\sum_{a} P(a)\left\langle I\left|V^{(4)}\right| J\right\rangle^{(a)},
$$

where $\Sigma_{a}=\sum_{a_{4}=1}^{4} \sum_{a_{3}=1}^{3} \sum_{a_{2}=1}^{2}$, and

$$
\begin{aligned}
\left\langle I\left|V^{(4)}\right| J\right\rangle^{(a)}= & \sum_{l, \tau} v_{l}^{s, j_{a 4}-f, j_{a 3}} c_{l, \tau}\left(i^{s} j_{a 4}\right) c_{l, \tau}\left(i_{3}^{f} j_{a 3}\right) \\
& \times \delta\left(i_{2}^{f}, j_{a 2}\right) \delta\left(i_{1}^{f}, j_{a 1}\right)+p . i .
\end{aligned}
$$

Here, p.i. stands for the other pair Coulomb interactions, Eq. (2.12). [The explicitly written term in Eq. (2.14) corresponds to the interaction between the fourth and third electron.] The elements $c_{\Lambda}(i j) \equiv c_{l, \tau}(i j)$ are defined by

$$
c_{\Lambda}(i j)=\int d \Omega\langle i \mid \hat{n}\rangle S_{\Lambda}(\hat{n})\langle\hat{n} \mid j\rangle .
$$

For the $7 s 5 f^{3}$ configuration there are three types of these coefficients. For the $s-s$ transition it is only one integral $\left\langle s\left|Y_{0}^{0}\right| s\right\rangle=1 / \sqrt{4 \pi}$, which is not zero. For the $f$ - $f$ transitions and real spherical harmonics, ${ }^{16}$ the coefficients $c_{l, \tau}\left(i^{f} j^{f}\right)$ were tabulated in Ref. 1. Finally, there are $f-s$ and $s-f$ transitions which require the evaluation of $c_{l, \tau}\left(i^{f} j^{s}\right)$. From the orthogonality of spherical harmonics, we find that

$$
\left\langle 0,0\left|Y_{3}^{\tau}\right| 3, \tau\right\rangle=\frac{1}{\sqrt{4 \pi}},
$$

where $\tau=0,(m, c),(m, s), m=1-3$, and zero otherwise. The matrix quantities (2.15) were first introduced by Condon and Shortley for the description of atomic spectra, ${ }^{5}$ but they are also at the center of the calculation of the crystal electric field effects. ${ }^{1}$

In Eq. (2.14) $v_{l}^{s, j_{a 4}-f, j_{a 3}}$ stands for a radial average. The general expression is

$$
\begin{aligned}
v_{l}^{a, b-c, e}= & \int d r r^{2} \int d r^{\prime} r^{\prime 2} \mathcal{R}_{a}(r) \mathcal{R}_{b}(r) \\
& \times \mathcal{R}_{c}\left(r^{\prime}\right) \mathcal{R}_{e}\left(r^{\prime}\right) v_{l}\left(r, r^{\prime}\right),
\end{aligned}
$$

where $\mathcal{R}_{a}, \mathcal{R}_{b}, \mathcal{R}_{c}$, and $\mathcal{R}_{e}$ are radial components, and $v_{l}\left(r, r^{\prime}\right)=v_{\Lambda \Lambda}\left(r, r^{\prime}\right)$ is given by Eq. (2.10). For the $7 s 5 f^{3}$ configuration the indices $a, b, c, e$ refer either to $7 s$ or to $5 f$ electron radial components. There are only two types of radial integrals, corresponding to nontrivial multipolar terms $(l \neq 0)$ of $7 s 5 f^{3}$, which are

$$
\begin{gathered}
v_{l}^{f f-f f}=\int d r r^{2} \int d r^{\prime} r^{\prime 2} \mathcal{R}_{f}^{2}(r) \mathcal{R}_{f}^{2}\left(r^{\prime}\right) v_{l}\left(r, r^{\prime}\right), \\
v_{l^{\prime}}^{f s-s f}=\int d r r^{2} \int d r^{\prime} r^{\prime 2} \mathcal{R}_{s}(r) \mathcal{R}_{f}(r) \\
\times \mathcal{R}_{f}\left(r^{\prime}\right) \mathcal{R}_{s}\left(r^{\prime}\right) v_{l}\left(r, r^{\prime}\right) .
\end{gathered}
$$

Here, $l=2,4,6$ and $l^{\prime}=3$, as follows from the selection rules for $c_{\Lambda}(f f)$ and $c_{\Lambda^{\prime}}(f s)$. The radial integrals $v_{l}^{f f-f f}$ and $v_{l^{\prime}}^{f s-s f}$ are proportional to the quantities $F_{l}$ and $G_{l}$ introduced by Condon and Shortley in Ref. 5. It is important to notice that even the $7 s$ electron with the trivial dependence of its angular part is strongly coupled to the three $5 f$ electrons through $f-s$ and $s-f$ transitions.

The classification scheme for electronic transitions, which we have introduced here, is very useful for handling the single particle interactions as well. The main difference is that now the interaction occurs with a single electron while the rest of them produce Kronecker factors, Ref. 3. This group of interactions includes the spin-orbit coupling $H_{s o}$, the crystal electric field $V_{C F}$, and the mean field $V_{M F}$. The latter two interactions are dealt with in Secs. III and IV. The spin-orbit coupling is

$$
H_{s o}=\sum_{i} V_{s o}(i),
$$

where the sum runs over all electrons, $V_{\text {so }}$ being the corresponding one-electron spin-orbit operator. The $s$ electron does not experience the spin-orbit coupling and, in the $7 s 5 f^{3}$ case, the summation includes only three $5 f$ terms $V_{s o}\left(i^{f}\right), i^{f}$ $=1-3$, where

$$
V_{s o}\left(i^{f}\right)=\zeta_{f} \vec{L}\left(i^{f}\right) \vec{S}\left(i^{f}\right) .
$$

Here, $\vec{L}\left(i^{f}\right)$ and $\vec{S}\left(i^{f}\right)$ are the one-electron operators of orbital and spin momentum; $\zeta_{f}$ is the constant of the spin-orbit coupling. The full intrasite Hamiltonian is given by $H_{\text {intra }}=V^{(4)}$ $+H_{s o}$. It describes the $7 s 5 f^{3}$ configuration of a free neptunium ion. Since the present method is not based on perturbation theory, it extends the classical calculations of Condon and Shortley. 5

\section{E. Intersite matrix elements}

We start with expression (2.8) and write it in the space of many-electron basis vectors $|I\rangle$, Eq. (2.2). Carrying out the angular integrations $d \Omega(\vec{n}), d \Omega^{\prime}(\vec{n}), d \Omega\left(\vec{n}^{\prime}\right), d \Omega^{\prime}\left(\vec{n}^{\prime}\right)$, we obtain

$$
\begin{aligned}
&\left\langle\left. I\right|_{\vec{n}}\left\langle\left. I^{\prime}\right|_{\vec{n}^{\prime}} V\left(\vec{R}(\vec{n}), \vec{R}^{\prime}\left(\vec{n}^{\prime}\right)\right) \mid J^{\prime}\right\rangle_{\vec{n}^{\prime}} \mid J\right\rangle_{\vec{n}^{\prime}} \\
&= \frac{1}{N_{a}} \sum_{a(\vec{n})} \sum_{a^{\prime}\left(\vec{n}^{\prime}\right)} P\left(a_{\vec{n}}\right) P\left(a_{\vec{n}^{\prime}}^{\prime}\right) \sum_{\alpha=1}^{4} \sum_{\alpha^{\prime}=1}^{4} \sum_{\Lambda \Lambda^{\prime}} v_{\Lambda}^{\alpha \alpha \alpha^{\prime} \alpha^{\prime}}\left(\vec{n}-\vec{n}^{\prime}\right) \\
& \times\left\{\begin{array}{c}
\left.c_{\Lambda}\left(i_{\alpha j} j_{\alpha}^{a}\right) \prod_{\beta=1}^{3} \delta\left(i_{\beta} j_{\beta}^{a}\right)\right\} \\
\left.c_{\Lambda^{\prime}}\left(i_{\alpha^{\prime}}^{\prime} j^{\prime a^{\prime}} \alpha^{\prime}\right) \prod_{\beta^{\prime}=1}^{3} \delta\left(i_{\beta^{\prime}}^{\prime} j^{\prime a^{\prime}} \beta^{\prime}\right)\right\}
\end{array}\right.
\end{aligned}
$$

Here, the sum over $a$ means the summation over all permutations of indices $j_{1}^{f}, j_{2}^{f}, j_{3}^{f}, j^{s}$ at site $\vec{n}$ transforming them to indices $j_{\alpha}^{a}(\alpha=1-4)$. Analogously, the sum over $a^{\prime}$ implies the summation over all permutations of $j_{1}^{\prime f}, j_{2}^{\prime f}, j_{3}^{\prime f}, j^{\prime s}$ at site $\vec{n}^{\prime}$ transforming them to $j_{\alpha}^{\prime a^{\prime}}(\alpha=1-4) . P(a)$ and $P\left(a^{\prime}\right)$ stand for the parities of the permutations. Indices $\alpha$ and $\alpha^{\prime}$ indicate which electron at site $\vec{n}$ interacts with which electron at site $\vec{n}^{\prime}$. The other electrons labeled by $\beta=1-3$ at site $\vec{n}$ and by $\beta^{\prime}=1-3$ at site $\vec{n}^{\prime}$ do not contribute to the interaction and 
TABLE II. The nine lowest and two highest eigenvalues of $5 f^{3}$, calculated with $v_{l}^{F-F}$, Eq. (3.3). Here, $g$ stands for the Landé factor; $E^{A}$ refers to the calculation of Amoretti et al., Ref. 20. Two highest values of $E^{A}$ marked by asterisk were reproduced by our calculation with the parameters of Ref. 20 .

\begin{tabular}{cccccc}
\hline \hline & Term & Deg. & $g\left(\mu_{B}\right)$ & $E, \mathrm{meV}$ & $E^{A}, \mathrm{meV}$ \\
\hline 1 & ${ }^{4} I_{9 / 2}$ & 10 & 0.7546 & 0 & 0 \\
2 & ${ }^{4} I_{11 / 2}$ & 12 & 0.9704 & 635.3 & 657.7 \\
3 & ${ }^{4} I_{13 / 2}$ & 14 & 1.0993 & 1204.2 & 1256.2 \\
4 & ${ }^{4} F_{3 / 2}$ & 4 & 0.6027 & 1244.2 & 948.3 \\
5 & ${ }^{2} H_{9 / 2}$ & 10 & 1.0154 & 1617.7 & 1438.3 \\
6 & ${ }^{4} F_{5 / 2}$ & 6 & 1.0067 & 1702.9 & 1399.3 \\
7 & ${ }^{4} I_{15 / 2}$ & 16 & 1.1797 & 1715.3 & 1762.3 \\
8 & ${ }^{4} S_{3 / 2}$ & 4 & 1.6546 & 1861.6 & 1614.9 \\
9 & ${ }^{4} F_{7 / 2}$ & 8 & 1.1195 & 1955.3 & 1697.1 \\
$\ldots$ & $\ldots$ & $\ldots$ & $\ldots$ & $\ldots$ & $\ldots$ \\
40 & ${ }^{2} F_{7 / 2}$ & 8 & 1.1317 & 7796.6 & $6541.1^{*}$ \\
41 & ${ }^{2} F_{5 / 2}$ & 6 & 0.8589 & 8008.2 & $6631.6^{*}$ \\
\hline
\end{tabular}

produce the Kronecker delta symbols. The coefficients $c_{\Lambda}$ are defined by Eq. (2.15), and the intersite $\left(\vec{n} \neq \vec{n}^{\prime}\right)$ interaction element $v_{\Lambda \Lambda^{\prime}}^{\alpha \alpha \alpha^{\prime} \alpha^{\prime}}$ is given by

$$
\begin{aligned}
v_{\Lambda \Lambda^{\prime}}^{\alpha \alpha \alpha^{\prime} \alpha^{\prime}}\left(\vec{n}-\vec{n}^{\prime}\right)= & \int d r r^{2} \int d r^{\prime} r^{\prime 2} \mathcal{R}_{\alpha}^{2}(r) \\
& \times \mathcal{R}_{\alpha^{\prime}}^{2}\left(r^{\prime}\right) v_{\Lambda \Lambda^{\prime}}\left(\vec{n}, \vec{n}^{\prime} ; r, r^{\prime}\right) .
\end{aligned}
$$

For the four-electron space of $7 s 5 f^{3}$, only even $l$ and $l^{\prime}$ (in $\Lambda$, $\Lambda^{\prime}$ ) are retained in Eqs. (2.21) and (2.22), and $l, l^{\prime}=0,2,4,6$. Two very important examples of intersite Coulomb interactions, namely, the crystal electric field and the mean field, will be considered in Secs. III and IV.

\section{CRYSTAL AND MEAN FIELD OF THE $5 f^{3}$ CONFIGURATION}

In this section we study the model where we assume that there are only three localized $5 f$ electrons at each neptunium site. ${ }^{19}$ Although we believe that the model is not adequate for a realistic description of $\mathrm{NpO}_{2}$, especially the part concerning the loss of the magnetic moments in the ordered phase, it is nevertheless very instructive to consider it in detail. The $5 f^{3}$ configuration being relatively simple offers an opportunity for thorough study and to understand the interplay between the disordered and ordered phases, or between the crystal and quadrupolar mean field. On the other hand, the configurations $7 p 5 f^{3}$ and $6 d 5 f^{3}$ involve too many basis states and consume too much time to be processed self-consistently for any temperature.

\section{A. Free-ion electron energy spectrum}

The basis states for the $5 f^{3}$ configuration are given by

$$
|I\rangle=\left|i_{1}^{f}, i_{2}^{f}, i_{3}^{f}\right\rangle,
$$

where as before, $i^{f}=1-14$. The total number of basis vectors is $14 \times 13 \times 12 / 3 !=364$. We treat the $5 f^{3}$ configuration in the way which was specified in Sec. II.
There are only $f-f$ transitions described by four radial integrals (2.18a): $v_{l}^{f f-f f}, l=0,2,4,6$. The others are zero due to the selection rules imposed by the coefficients $c_{\Lambda}(i j)$, Eq. (2.15). The radial integral $v_{0}^{f f-f f}$ (Hubbard $U$ ) is not important here since it does not result in term splittings. In the following we will use the condensed notation $F$ for $f f$, and thus $v_{l}^{f f-f f} \equiv v_{l}^{F-F}$. These quantities are connected with the Slater (Condon-Shortley) parameters ${ }^{5} F^{l}(5 f, 5 f)$ through the following relation:

$$
v_{l}^{F-F}=\frac{4 \pi}{2 l+1} F^{l} .
$$

In particular, the Slater parameters $F^{2}, F^{4}$, and $F^{6}$ of Amoretti et al. ${ }^{20}$ correspond to $v_{2}^{F-F}=14.007 \mathrm{eV}, v_{4}^{F-F}=7.091 \mathrm{eV}$, and $v_{6}^{F-F}=3.168 \mathrm{eV}$. (In order to obtain the exact term splitting quoted in Table II of Ref. 20, we had to scale their Slater parameters by a factor of 0.9755 .) Alternatively, the quantities $v_{l}^{F-F}$ can be calculated by using the radial dependence of the $5 f$ electrons $\mathcal{R}_{f}$, Eq. (2.18a). We have done such a calculation and then corrected the parameters by comparing the splittings of the $f^{3}$ configuration with experimental data for $\mathrm{Pr}^{3+}$ and $\mathrm{Nd}^{4+}$ (details are given in the Appendix). We arrived at

$$
\begin{gathered}
v_{2}^{F-F}=18.164, \quad v_{4}^{F-F}=8.578, \\
v_{6}^{F-F}=3.362, \quad \zeta_{f}=0.2547, \quad \text { in } \mathrm{eV} .
\end{gathered}
$$

After calculating the matrix elements of the Coulomb repulsion and the spin-orbit coupling, we diagonalize the matrix

$$
H_{\text {intra }}=V^{(3)}+H_{\text {so }},
$$

and obtain the electronic spectrum of $5 f^{3}$. The nine lowest and two highest eigenvalues are shown in Table II, where for comparison we also quote the spectrum of free $\mathrm{Np}$ ion of Ref. 20. Notice that, in comparison with the spectrum ${ }^{20}$ of Amoretti et al., ${ }^{4} F_{3 / 2}$ and ${ }^{4} F_{5 / 2}$ are higher than ${ }^{4} I_{13 / 2}$ and 
${ }^{2} H_{9 / 2}$, which is in better agreement with the sequence of terms for the $4 f^{3}$ configurations of $\mathrm{Pr}^{3+}$ and $\mathrm{Nd}^{4+}$ known from experiment. ${ }^{10}$

\section{B. Crystal electric field (CEF) excitations in the disordered phase $(T>25 \mathrm{~K})$}

In the disordered (no quadrupole electron density) phase $(T>25 \mathrm{~K})$ the electron density of the $5 f^{3}$ configuration adopts the cubic $\left(O_{h}\right)$ site symmetry. This density modulation is induced by the cubic crystal electric field experienced by three $5 f$ electrons. In terms of the multipole intersite expansion (2.8) it implies that, for a given Np site $\vec{n}$, we treat 12 Np neighbors $\left(\vec{n}_{1}^{\prime}=1-12\right)$ and 8 oxygen neighbors $\left(\vec{n}_{2}^{\prime}\right.$ $=1-8)$ in spherical approximation, i.e., $l^{\prime}=0$ and $S_{\Lambda^{\prime}}\left(\vec{n}^{\prime}\right)$ reduces to $Y_{0}^{0}=1 / \sqrt{4 \pi}$. The coefficients $c_{\Lambda^{\prime}}$, Eq. $(2.15)$, become simple

$$
c_{0}\left(i_{\alpha^{\prime}} j_{\alpha^{\prime}}\right)=\frac{1}{\sqrt{4 \pi}} \delta\left(i_{\alpha^{\prime}}, j_{\alpha^{\prime}}\right) .
$$

Here, we write 0 for $\Lambda^{\prime} \equiv\left(l^{\prime}=0, A_{1 g}\right)$. At the central site $\vec{n}$ we expand the CEF in terms of SAFs $S_{\Lambda_{1}}(\vec{n}), \Lambda_{1} \equiv\left(l, A_{1 g}\right)$, where $A_{1 g}$ stands for the unit representation of the cubic site group $O_{h}$. The selection rules for the coefficients $c_{\Lambda}\left(i_{\alpha}, j_{\alpha}\right)$ of the $f-f$ transitions imply that there remain only two nontrivial functions $S_{\Lambda_{1}}$ with $l=4$ and $l=6$, which correspond to the cubic harmonics $K_{4}(\Omega)$ and $K_{6}(\Omega)$. The multipole two-center expansion (2.8) becomes

$$
V\left(\vec{R}(\vec{n}), \vec{R}^{\prime}\left(\vec{n}^{\prime}\right)\right)=\frac{1}{\sqrt{4 \pi}} \sum_{\Lambda_{1}} v_{\Lambda_{1} 0}\left(\vec{n}, \vec{n}^{\prime} ; r, r^{\prime}\right) S_{\Lambda_{1}}(\vec{n}),
$$

where

$$
\begin{aligned}
& v_{\Lambda_{1} 0}\left(\vec{n}, \vec{n}^{\prime} ; r, r^{\prime}\right) \\
& \quad=\frac{1}{\sqrt{4 \pi}} \int d \Omega(\vec{n}) \int d \Omega^{\prime}\left(\vec{n}^{\prime}\right) \frac{S_{\Lambda_{1}}(\hat{n})}{\left|\vec{R}(\vec{n})-\vec{R}^{\prime}\left(\vec{n}^{\prime}\right)\right|} .
\end{aligned}
$$

Here, $v_{\Lambda_{1} 0}\left(\vec{n}, \vec{n}^{\prime} ; r, r^{\prime}\right)$ has the same value for all $12 \mathrm{~Np}$ neighbors $\left(\vec{n}_{1}^{\prime}=1-12\right)$, and a same value for all 8 oxygen neighbors $\left(\vec{n}_{2}^{\prime}=1-8\right)$. As follows from Eq. (2.11), $v_{\Lambda_{1} 0}\left(\vec{n}, \vec{n}^{\prime} ; r, r^{\prime}\right)$ is independent of $r^{\prime}$. Equation (2.22) then can be written in the following form:

$$
v_{\Lambda_{1} 0}^{\alpha \alpha \alpha^{\prime} \alpha^{\prime}}\left(\vec{n}-\vec{n}^{\prime}\right)=v_{\Lambda_{1} 0}^{\alpha \alpha} \times Q_{\alpha^{\prime}},
$$

where

$$
v_{\Lambda_{1} 0}^{\alpha \alpha}=\int d r r^{2} \mathcal{R}_{\alpha}^{2}(r) v_{\Lambda_{1} 0}\left(\vec{n}, \vec{n}^{\prime} ; r, r^{\prime}\right)
$$

and

$$
Q_{\alpha^{\prime}}=\int d r^{\prime} r^{\prime 2} \mathcal{R}_{\alpha^{\prime}}^{2}\left(r^{\prime}\right) .
$$

Here, the integrations are taken over $0<r^{\prime}<R_{M T}$, where $R_{M T}$ is the radius of the muffin-tin sphere of neptunium or
TABLE III. Calculated parameters of the CEF. $v_{l}^{N p N p}$ $=v_{\Lambda_{1} 0}\left(\vec{n}, \vec{n}_{1}^{\prime} ; R_{M T}^{N p}, r^{\prime}\right), \vec{n}=0$ is the central Np site, $\vec{n}_{1}^{\prime}$ is one of $12 \mathrm{~Np}$ nearest neighbors. $v_{l}^{N p O}{ }_{0}=v_{\Lambda_{1} 0}\left(\vec{n}, \vec{n}_{2}^{\prime} ; R_{M T}^{N p}, r^{\prime}\right), \vec{n}_{2}^{\prime}$ is one of six oxygen neighbors.

\begin{tabular}{cccc}
\hline \hline & Units & $l=4$ & $l=6$ \\
\hline$v_{l}^{N p O}$ & $\mathrm{meV}$ & -816.7 & 209.7 \\
$v_{l}^{N p N p}$ & $\mathrm{meV}$ & -26.37 & -6.190 \\
$q_{l}^{f} /\left(R_{M T}^{N p}\right)^{l}$ & & 0.1592 & 0.0994 \\
$B_{l}^{O} / Q_{e f f}^{O} e$ & $\mathrm{~K}$ & -3405.2 & 546.0 \\
$B_{l}^{N p} / Q_{\text {eff }}^{N p} e$ & $\mathrm{~K}$ & -165.0 & -24.2 \\
\hline \hline
\end{tabular}

oxygen. (The influence of the interstitial region will be discussed later.) $Q_{\alpha^{\prime}}$ refers to an electron at site $\vec{n}^{\prime}$ which interacts with one of the three $5 f$ electrons at $\vec{n}$. We then can perform a summation over all electrons at $\vec{n}^{\prime}$ and include also in this term the interaction with the nucleus. This results in replacing $Q_{\alpha^{\prime}}$ by $e Q_{M T}$ in Eq. (3.7), $Q_{M T}$ and $e$ being the total charge inside the MT sphere and the electron charge $(e=-1)$, respectively. From Eq. (2.11), it follows that

$$
v_{\Lambda_{1} 0}^{\alpha \alpha}=v_{\Lambda_{1} 0}\left(\vec{n}, \vec{n}^{\prime} ; R_{M T}, r^{\prime}\right) \frac{q_{l}^{\alpha}}{R_{M T}^{l}},
$$

where $l$ in the index $\Lambda_{1}$ is 4 or 6 , and

$$
q_{l}^{\alpha}=\int d r^{\prime} r^{\prime(l+2)} \mathcal{R}_{\alpha}^{2}\left(r^{\prime}\right) .
$$

Therefore, the CEF operator for any neptunium site $(r$ $<R_{M T}^{N p}$ ) can be written explicitly as

$$
V_{C F}(\vec{R}(\vec{n}))=\sum_{l=4,6} B_{l} S_{\left(l, A_{1 g}\right)}(\hat{n})\left(\frac{r}{R_{M T}^{N p}}\right)^{l},
$$

where

$$
B_{l}=B_{l}^{N p}+B_{l}^{O}
$$

and

$$
\begin{aligned}
& B_{l}^{N p}=\frac{12}{\sqrt{4 \pi}} Q_{e f f}^{N p} e v_{\Lambda_{1} 0}\left(\vec{n}, \vec{n}_{1}^{\prime} ; R_{M T}^{N p}, r^{\prime}\right), \\
& B_{l}^{O}=\frac{8}{\sqrt{4 \pi}} Q_{e f f}^{O} e v_{\Lambda_{1} 0}\left(\vec{n}, \vec{n}_{2}^{\prime} ; R_{M T}^{N p}, r^{\prime}\right) .
\end{aligned}
$$

We quote all relevant parameters of $\mathrm{CEF}$ in Table III. As given by Eq. (3.10a), the CEF operator $V_{C F}$ is a one-electron quantity. ${ }^{21,22} \mathrm{CEF}$ acts along with the Coulomb intrasite repulsion, Eq. (3.4). Therefore, the total Hamiltonian for the disordered phase becomes

$$
H^{d i s}(\vec{n})=H_{\text {intra }}+V_{C F}(\vec{n}) .
$$

Although we have considered CEF from first principles, there is still an ambiguity related to the charge distribution in the interstitial region. A more rigorous treatment of the problem is given in Refs. 23 and 24. A careful consideration of the problem based on the solution of a periodic Poisson's 
equation leads to a renormalization of the charges inside the MT spheres, Ref. 24. In other words, in Eqs. (3.10a)-(3.10d) the effective charges for $\mathrm{Np}$ and $\mathrm{O}$ are given by

$$
Q_{e f f}=Q_{M T}-\frac{4 \pi R_{M T}^{3}}{3} \rho_{I}(\vec{K}=0)-4 \pi R_{M T}^{2} \sum_{\vec{K} \neq 0}{ }^{\prime} \frac{j_{1}\left(K R_{M T}\right)}{K} \rho_{I}(\vec{K}),
$$

where $\rho_{I}(\vec{K})$ is the Fourier series expansion of the electron density in the interstitial region; $j_{l=1}$ is the spherical Bessel function. $\rho_{I}(\vec{K}=0)$ is the average density in the interstitial region, $Q_{\text {out }} / V_{\text {out }}$, where $Q_{\text {out }}$ and $V_{\text {out }}$ are the charge and volume of the interstitial region, respectively.

The calculation of $Q_{\text {eff }}$ according to Eq. (3.12) is quite laborious since it requires the evaluation of the Fourier coefficients $\rho_{I}(\vec{K})$. Instead, below we consider two approximations to (3.12). In the first approximation we assume that $Q_{e f f}^{N p}(I)=Q_{M T}^{N p}$ and $Q_{e f f}^{O}(I)=Q_{M T}^{O}$, where $Q_{M T}^{N p}$ and $Q_{M T}^{O}$ are the total charges inside the MT spheres of neptunium and oxygen. Here, the electron charge in the interstices is completely ignored. In the second approximation we take

$$
Q_{e f f}(I I)=Q_{M T}-\frac{4 \pi R_{M T}^{3}}{3} \rho_{I}(\vec{K}=0) .
$$

This expression corresponds to the homogeneous electron density distribution in the interstitial region. However, the modification of effective charges in this approximation is too strong: $Q_{e f f}^{N p}(I)=+4.108|e|$ and $Q_{e f f}^{N p}(I I)=+5.337|e|$ for neptunium, $Q_{e f f}^{O}(I)=-0.284|e|$ and $Q_{e f f}^{O}(I I)=+0.945|e|$ for oxygen. As was discussed in Ref. 2, the CEF splitting is overestimated in the second approximation. In reality the charge density in interstices is highly inhomogeneous, concentrated mainly in the proximity to oxygen and neptunium. This leads to $\rho_{I}(\vec{K} \neq 0) \neq 0$, and the last term in Eq. (3.12) acts in the opposite direction, decreasing $Q_{\text {eff }}$ backward to $Q_{M T}$ values, which correspond to the first approximation.

The exact calculation of $Q_{\text {eff }}$ according to Eq. (3.12) is beyond the scope of the present study. Instead, we have studied the crystal field effects as a function of $Q_{\text {eff }}$ by introducing

$$
\begin{gathered}
Q_{e f f}^{N p}\left(x_{e f f}\right)=Q_{M T}^{N p}+x_{e f f}\left(Q_{e f f}^{N p}(I I)-Q_{M T}^{N p}\right), \\
Q_{e f f}^{O}\left(x_{e f f}\right)=Q_{M T}^{O}+x_{e f f}\left(Q_{e f f}^{O}(I I)-Q_{M T}^{O}\right),
\end{gathered}
$$

where $0<x_{\text {eff }}<1$. Diagonalizing $H^{\text {dis }}$, Eq. (3.11), we have found that 41 terms of $5 f^{3}$ are split into 120 distinct sublevels of $\Gamma_{6}, \Gamma_{7}$, and $\Gamma_{8}$ symmetry of the cubic double-group $O_{h}^{\prime}$. In particular, two lowest atomic-like levels are split according to the following scheme:

$$
\begin{gathered}
{ }^{4} I_{9 / 2} \rightarrow \Gamma_{8}+\Gamma_{8}+\Gamma_{6}, \\
{ }^{4} I_{11 / 2} \rightarrow \Gamma_{8}+\Gamma_{7}+\Gamma_{6}+\Gamma_{8} .
\end{gathered}
$$

The resulting splittings and the dependence of CEF on $x_{\text {eff }}$ are shown in Fig. 1. The splittings of two lowest terms of the $5 f^{3}$ configuration, Eqs. (3.15a) and (3.15b), is also given in Tables IV and $\mathrm{V}$ for $x_{e f f}=0$ and $x_{e f f}=0.5$, respectively.

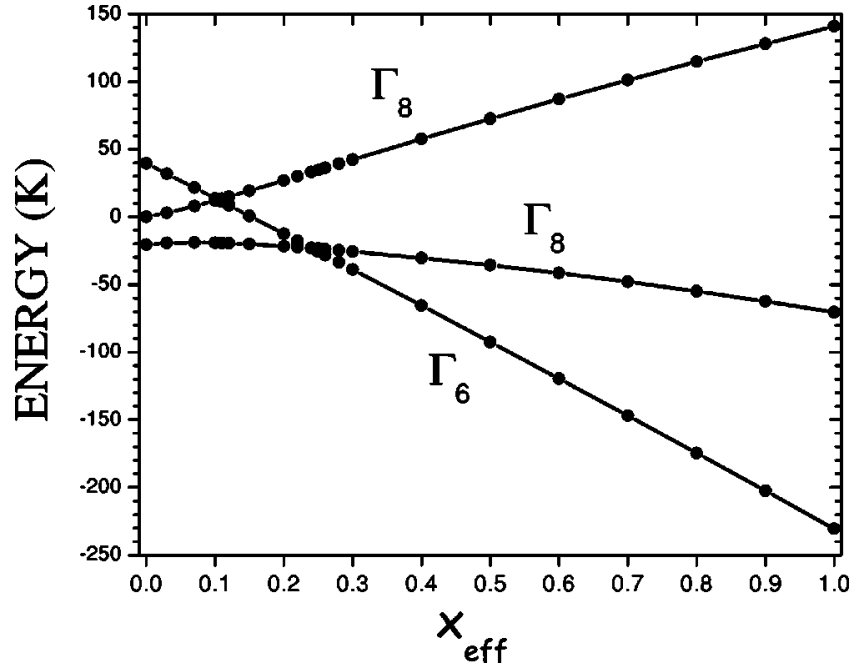

FIG. 1. Splitting of the lowest ${ }^{4} I_{9 / 2}$ terms of the $5 f^{3}$ configuration of $\mathrm{Np}$ in cubic crystal field, Eqs. (3.10a)-(3.10d) as a function of the effective charges of neptunium and oxygen, Eqs. (3.14a) and (3.14b). Zero corresponds to the energy of the ${ }^{4} I_{9 / 2}$ level of free $\mathrm{Np}$ ion.

The most comprehensive study of the crystal field of the $5 f^{3}$ configuration was performed by Amoretti et al., Ref. 20. Comparing their results with ours, we obtain the following relations connecting $B_{4}$ and $B_{6}$ with $V_{4}$ and $V_{6}$ used there:

$$
\begin{gathered}
B_{4}=8 \sqrt{\frac{12}{7}} V_{4}, \\
B_{6}=16 \sqrt{8} V_{6} .
\end{gathered}
$$

We observe that, for a realistic choice of $Q_{e f f}$, which corresponds to $x_{e f f} \sim 0-0.5$, the CEF splitting is a few times smaller than the value of $55 \mathrm{meV}$ considered for CEF excitations in Ref. 20. Correspondingly, the calculated parameters $B_{4}$ and $B_{6}$ (Tables IV and V) are smaller. Notice that it is not possible to relate the feature at $55 \mathrm{meV}$ with the ${ }^{4} I_{11 / 2}$ splittings because it is situated at much higher energy $\sim 650 \mathrm{meV}$, Table II. Most likely, the experimental excitations at $55 \mathrm{meV}$ refer to the valence electrons delocalized on the $\mathrm{Np}-\mathrm{O}$ bonds, while the lowest $\mathrm{CEF}$ excitations of $\mathrm{Np}$ lie

TABLE IV. CEF low energy spectrum and magnetic moments of the $5 f^{3}$ configuration of $\mathrm{Np} ; x_{e f f}=0, \Delta \epsilon=7380.7 \mathrm{~K}$. Calculated CEF parameters $B_{4}=-288.1 \mathrm{~K}, B_{6}=254.2 \mathrm{~K}$, Eq. (3.10a).

\begin{tabular}{ccccc}
\hline \hline & $\Gamma$ & Deg. & $\left(\epsilon_{i}-\epsilon_{1}\right)(\mathrm{K})$ & $\mathcal{M}_{z}\left(\mu_{B}\right)$ \\
\hline${ }^{4} I_{9 / 2}$ & $\Gamma_{8}$ & 4 & 0 & $\pm(1.275,1.429)$ \\
& $\Gamma_{8}$ & 4 & 20.5 & $\pm(0.517,1.686)$ \\
& $\Gamma_{6}$ & 2 & 60.2 & \pm 1.384 \\
${ }^{4} I_{11 / 2}$ & $\Gamma_{6}$ & 2 & $\Delta \epsilon$ & \pm 1.778 \\
& $\Gamma_{8}$ & 4 & $\Delta \epsilon+4.6$ & $\pm(1.241,2.120)$ \\
& $\Gamma_{7}$ & 2 & $\Delta \epsilon+6.3$ & \pm 1.775 \\
& $\Gamma_{8}$ & 4 & $\Delta \epsilon+26.1$ & $\pm(1.119,3.835)$ \\
\hline \hline
\end{tabular}


TABLE V. CEF low energy spectrum and magnetic moments of the $5 f^{3}$ configuration of $\mathrm{Np} ; x_{e f f}=0.5, \Delta \epsilon=7396.3 \mathrm{~K}$. Calculated CEF parameters $B_{4}=1905.7 \mathrm{~K}, B_{6}=-66.5 \mathrm{~K}$, Eq. (3.10a).

\begin{tabular}{ccccc}
\hline \hline & $\Gamma$ & Deg. & $\left(\epsilon_{i}-\epsilon_{1}\right)(\mathrm{K})$ & $\mathcal{M}_{z}\left(\mu_{B}\right)$ \\
\hline${ }^{4} I_{9 / 2}$ & $\Gamma_{6}$ & 2 & 0 & \pm 1.383 \\
& $\Gamma_{8}$ & 4 & 56.8 & $\pm(0.451,2.077)$ \\
& $\Gamma_{8}$ & 4 & 164.9 & $\pm(1.204,2.333)$ \\
${ }^{4} I_{11 / 2}$ & $\Gamma_{8}$ & 4 & $\Delta \epsilon$ & $\pm(0.392,3.862)$ \\
& $\Gamma_{7}$ & 2 & $\Delta \epsilon+28.6$ & \pm 1.777 \\
& $\Gamma_{8}$ & 4 & $\Delta \epsilon+110.8$ & $\pm(1.290,2.845)$ \\
& $\Gamma_{6}$ & 2 & $\Delta \epsilon+132.6$ & \pm 1.785 \\
\hline \hline
\end{tabular}

at a smaller energy $\sim 6 \mathrm{meV}$, Tables IV and V.

Finally, we would like to note that our CEF calculation is based on first principles, and in that respect it differs from the others, ${ }^{20,25,26}$ which use fitting from experiment to extract the CEF parameters. There are also other technical differences. In contrast to Refs. 25 and 26 we do not assume that the full momentum $J$ is a good quantum number, that allows for a mixing of components belonging to different $J$. In Ref. 20 the basis was truncated to the first 11 low-lying levels. In our approach we do not have these limitations.

\section{Mean field and the structural phase transition at $25 \mathrm{k}$}

Recent resonant X-ray scattering (RXS) experiments at the $\mathrm{Np} M_{I V}$ and $M_{V}$ edges in $\mathrm{NpO}_{2}$ indicated an unexpected result: the phase transition of $\mathrm{NpO}_{2}$ is not isostructural. ${ }^{13}$ In the low temperature phase a long-range order of $\mathrm{Np}$ electric quadrupoles was revealed by the growth of superlattice Bragg peaks. $^{13,27}$ The space symmetry of the ordered phase was identified as $P n 3 m .^{13}$ In real space the $P n \overline{3} m$ ordering is characterized by four different sublattices of the simple cubic structure. We label these sublattices which contain the sites $(0,0,0),(a / 2)(0,1,1),(a / 2)(1,0,1)$, and $(a / 2)(1,1,0)$ by $\left\{\vec{n}_{p}\right\}, p=1-4$, respectively. The most significant feature of the ordered phase is the existence of only one threefold axis of symmetry $C_{3}$ at each $\mathrm{Np}$ site which is also a cube diagonal. The only quadrupole function compatible with the symmetry lowering is $Y_{2}^{0}\left(\Omega^{\prime}\right)$ in the coordinate system where the $z^{\prime}$ axis coincides with one of the threefold axes (cube diagonals): [ $\left.\begin{array}{lll}1 & 1 & 1\end{array}\right],[-1,-1,1],[1,-1,-1]$, and $[-1,1,-1]$. Consequently, there are four such functions which are given by $^{28}$

$$
\begin{gathered}
\mathcal{S}_{a}(\Omega)=\frac{1}{\sqrt{3}}\left(Y_{2}^{1 s}(\Omega)+Y_{2}^{1 c}(\Omega)+Y_{2}^{2 s}(\Omega)\right), \\
\mathcal{S}_{b}(\Omega)=\frac{1}{\sqrt{3}}\left(-Y_{2}^{1 s}(\Omega)-Y_{2}^{1 c}(\Omega)+Y_{2}^{2 s}(\Omega)\right), \\
\mathcal{S}_{c}(\Omega)=\frac{1}{\sqrt{3}}\left(Y_{2}^{1 s}(\Omega)-Y_{2}^{1 c}(\Omega)-Y_{2}^{2 s}(\Omega)\right)
\end{gathered}
$$

$$
\mathcal{S}_{d}(\Omega)=\frac{1}{\sqrt{3}}\left(-Y_{2}^{1 s}(\Omega)+Y_{2}^{1 c}(\Omega)-Y_{2}^{2 s}(\Omega)\right)
$$

The real spherical harmonics ${ }^{16} Y_{2}^{1 s}, Y_{2}^{1 c}, Y_{2}^{2 s}$ belong to a three-dimensional irreducible representation $T_{2 g}$ of $O_{h}$. They are proportional to the Cartesian components $y z, z x$, and $x y$. The $\operatorname{Pn} \overline{3} m$ structure is obtained when $\mathcal{S}_{a}$ corresponds to the first sublattice $\left(\left\{\vec{n}_{1}\right\}\right), \mathcal{S}_{b}-\left\{\vec{n}_{4}\right\}, \mathcal{S}_{c}-\left\{\vec{n}_{2}\right\}$, and $\mathcal{S}_{d}-\left\{\vec{n}_{3}\right\} .{ }^{28}$

Now, we consider the intersite quadrupole interactions $V^{Q Q}\left(\vec{n}, \vec{n}^{\prime}\right)$ between a central Np site (sublattice $\left.\left\{n_{1}\right\}\right)$ and its 12 nearest $\mathrm{Np}$ neighbors belonging to sublattices $\left\{n_{p^{\prime}}\right\}, p^{\prime}$ $=2,3,4$. [Here, we will not take into account the interactions involving higher spherical harmonics because they are considerably smaller, Eq. (2.11).] This direct quadrupolequadrupole coupling can be calculated from first principles. Such a problem for the $P a \overline{3}$ structure has been considered in Refs. 1 and 2, where it is shown that the $P a \overline{3}$ spatial order of quadrupoles gives an effective attraction between them. For the $P n 3 m$ structure the direct interaction is repulsive, ${ }^{28}$ and we have to resort to an indirect coupling via oxygenmediated interaction as a driving force of the phase transition. The important fact which we exploit in this section is that, irrespective of the nature of the effective $\mathrm{Np}-\mathrm{Np}$ interaction, the mean field can be described by a general expression (3.24) below which simply reflects the rhombohedral (trigonal) symmetry of the neptunium site. ${ }^{13,28}$

We start by deriving an effective mean field for the direct electrostatic interaction between quadrupoles. Following Ref. 2, one obtains from Eq. (2.21) the following expression for the quadrupolar interaction operator between neptunium sites at $\vec{n}_{1}$ and $\vec{n}_{p^{\prime}}$ :

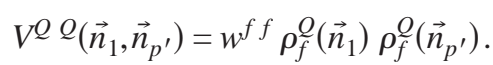

Here, $\vec{n}_{1} \in\left\{n_{1}\right\}, \vec{n}_{p^{\prime}} \in\left\{n_{p^{\prime}}\right\}$, and the quadrupolar density operator $\rho_{f}^{Q}$ at site $\vec{n}_{p}$ is given by

$$
\rho_{f}^{Q}(\vec{n})=\sum_{I, J}|I\rangle \sum_{a} P(a) \sum_{\alpha=1}^{3} c_{p}\left(i_{\alpha} j_{\alpha}^{a}\right) \prod_{\beta=1}^{2} \delta_{i_{\beta^{j}}{ }^{a}{ }_{\beta}\langle J|,}
$$

where again $a$ is a permutation of $j_{1}^{f}, j_{2}^{f}, j_{3}^{f}$ transforming them to a new order given by $j_{\kappa}^{a}, \kappa=1-3 . P(a)$ is the parity of the permutation; the index $\alpha$ stands for the interacting electron at the site. (The second interacting electron belongs to a neighboring $\mathrm{Np}$ site.) The other (noninteracting) electrons $(\beta=1,2)$ at $\vec{n}$ produce the product of the Kronecker delta symbols. The quadrupolar $f$ - $f$ coefficients are defined as

$$
c_{p}\left(i_{\alpha} j_{\alpha}\right)=\left\langle i_{\alpha}\left|\mathcal{S}_{p}\right| j_{\alpha}\right\rangle
$$

There are four types of such coefficients (i.e., $p=1-4)$ as follows from Eqs. (3.17a)-(3.17d). Finally, the interaction constant $w^{f f}$ in Eq. (3.18) is given by

$$
w^{f f}=\frac{1}{3}\left(\gamma^{f f}-2 \alpha^{f f}\right) .
$$

Here

$$
\gamma^{f f}=\int d r r^{2} \int d r^{\prime} r^{\prime 2} \mathcal{R}_{f}^{2}(r) \mathcal{R}_{f}^{2}\left(r^{\prime}\right) v_{\Lambda_{1} \Lambda_{1}}\left(\vec{n}, \vec{n}^{\prime} ; r, r^{\prime}\right),
$$


TABLE VI. Calculated parameters of the mean field.

\begin{tabular}{cccc}
\hline \hline$v_{11}^{Q Q}\left(R_{M T}^{N p}\right)$ & $v_{22}^{Q Q}\left(R_{M T}^{N p}\right)$ & $\gamma^{f f}$ & $\alpha^{f f}$ \\
\hline $4568.2 \mathrm{~K}$ & $-1442.6 \mathrm{~K}$ & $437.6 \mathrm{~K}$ & $-138.2 \mathrm{~K}$ \\
$q_{2}^{f} /\left(R_{M T}^{N p}\right)^{2}$ & $\lambda^{f f}(P n \overline{3} m)$ & $\lambda^{f f}(P a \overline{3})$ & \\
\hline 0.3095 & $2856 \mathrm{~K}$ & $-1750.5 \mathrm{~K}$ & \\
\hline \hline
\end{tabular}

$$
\alpha^{f f}=\int d r r^{2} \int d r^{\prime} r^{\prime 2} \mathcal{R}_{f}^{2}(r) \mathcal{R}_{f}^{2}\left(r^{\prime}\right) v_{\Lambda_{2} \Lambda_{2}}\left(\vec{n}, \vec{n}^{\prime} ; r, r^{\prime}\right),
$$

with $v_{\Lambda \Lambda}\left(\vec{n}, \vec{n}^{\prime} ; r, r^{\prime}\right)$ defined by Eq. (2.9), where $\vec{n}$ $=(0,0,0), \vec{n}^{\prime}=(a / 2)(0,1,1)$ and $\Lambda_{1}=\left(l=2, T_{2 g}, k=1\right), \Lambda_{2}$ $=\left(l=2, T_{2 g}, k=2\right)$. The corresponding $\mathrm{SAF}$ is $S_{\Lambda}=Y_{2}^{1 s}$. Using the property (2.11), we rewrite $\gamma^{f f}$ and $\alpha^{f f}$ as

$$
\begin{gathered}
\gamma^{f f}=\frac{q_{2}^{f}}{\left(R_{M T}^{N p}\right)^{2}} v_{11}^{Q Q}\left(R_{M T}^{N p}\right) \frac{q_{2}^{f}}{\left(R_{M T}^{N p}\right)^{2}}, \\
\alpha^{f f}=\frac{q_{2}^{f}}{\left(R_{M T}^{N p}\right)^{2}} v_{2}^{Q}{ }_{2}^{Q}\left(R_{M T}^{N p}\right) \frac{q_{2}^{f}}{\left(R_{M T}^{N p}\right)^{2}},
\end{gathered}
$$

where the short notation $v_{k k}^{Q Q}\left(R_{M T}^{N p}\right), k=1,2$, stands for the corresponding $v_{\Lambda \Lambda}\left(\vec{n}, \vec{n}^{\prime} ; R_{M T}^{N p}, R_{M T}^{N p}\right)$, and the "quadrupole charge" $q_{l=2}^{f}$ of $5 f$ electron is given by (3.9b).

In the mean-field approximation, after summing over 12 nearest neighbors belonging to three sublattices, we arrive at the effective bilinear quadrupole-quadrupole operator

$$
U^{Q Q}\left(\vec{n}_{p}\right)=\lambda^{f f}\left\langle\rho_{f}^{Q}\right\rangle \rho_{f}^{Q}\left(\vec{n}_{p}\right),
$$

where for $P n \overline{3} m$ the direct quadrupole-quadrupole interaction is repulsive, $\lambda^{f f}=4\left(\gamma^{f f}-2 \alpha^{f f}\right)>0$. The relevant calculated parameters are quoted in Table VI. (The same expression holds for $P a \overline{3}$, but in that case for the direct quadrupole interaction $\lambda^{f f}=4 \gamma^{f f}<0$, which means attraction.) $\left\langle\rho_{f}^{Q}\right\rangle$ stands for an expectation value of the quadrupole operator (3.19). At zero temperature it is the quantum average

$$
\left\langle\rho_{f}^{Q}\right\rangle=\left\langle I_{g s}\left|\rho_{f}^{Q}\left(\vec{n}_{p}\right)\right| I_{g s}\right\rangle,
$$

where $\left|I_{g s}\right\rangle$ refers to the ground state of the full mean-field Hamiltonian

$$
H^{M F}(\vec{n})=U^{Q Q}(\vec{n})+V_{C F}(\vec{n})+H_{\text {intra }}(\vec{n}) .
$$

The intrasite part of the interactions $H_{\text {intra }}$ is given by Eq. (3.4). For CEF we used the values $x_{e f f}=0$ and $Q_{e f f}^{N p}=Q_{M T}^{N p}$, $Q_{e f f}^{O}=Q_{M T}^{O}$. (As we discussed in Sec. III B, this gives the most realistic estimate for CEF.) If $\lambda^{f f}<0$ then at zero temperature the mean-field Hamiltonian $H^{M F}$ has a ground state with a nontrivial quadrupolar order parameter, $\left\langle\rho_{f}^{Q}\right\rangle \neq 0$. In such a case the site symmetry becomes trigonal (with the main point subgroup $C_{3}$ ) and the fourfold degeneracy of the former cubic quartet states is lifted
TABLE VII. Mean-field (trigonal) splittings at $T=0, \lambda_{\text {eff }} / \lambda^{f f}$ $=1 . T_{Q}=0.44 \mathrm{~K}, \rho_{0}=-0.0205$.

\begin{tabular}{ccccc}
\hline \hline$\Gamma$ & Deg. & $\left(\epsilon_{i}-\epsilon_{1}\right)(\mathrm{K})$ & $\mathcal{M}_{z}\left(\mu_{B}\right)$ & $\rho_{f}^{Q}$ \\
\hline$E$ & 2 & 0 & \pm 0.9464 & -0.0205 \\
$E$ & 2 & 1.1 & \pm 0.7962 & 0.0135 \\
$E$ & 2 & 20.7 & \pm 0.6406 & -0.0083 \\
$E$ & 2 & 21.7 & \pm 0.8428 & 0.0140 \\
$E$ & 2 & 60.8 & \pm 1.3840 & 0.0011 \\
\hline \hline
\end{tabular}

$$
\Gamma_{8} \rightarrow E+E,
$$

where $E$ stands for the twofold degenerate irreducible representation of $C_{3}$. The states $\Gamma_{6}$ remain unsplit as a consequence of the Kramers theorem, $\Gamma_{6} \rightarrow E$.

We now obtain a system of equations which can be solved self-consistently. At first we introduce an average $\left\langle\rho_{f}^{Q}\right\rangle$, which defines the interactions (3.24) and (3.26). In the space of the $5 f^{3}$ configuration (364 state vectors), we diagonalize the total Hamiltonian $H^{M F}$, Eq. (3.26), and obtain the eigenvectors $|K\rangle, K=1-364$

$$
H^{M F}|K\rangle=\epsilon_{K}|K\rangle,
$$

where the lowest value of $\epsilon_{K}$ corresponds to $K=1,2$. This is the Kramers doublet of the ground state. We then calculate the quantities

$$
\rho_{f}^{Q}(K)=\left\langle K\left|\rho_{f}^{Q}\left(\vec{n}_{p}\right)\right| K\right\rangle,
$$

which evaluate the quadrupolar moments of the states $K$. Next, we find an improved value for $\left\langle\rho_{f}^{Q}\right\rangle$ which is $\rho_{f}^{Q}(K$ $=1)=\rho_{f}^{Q}(K=2)$, Eq. (3.25). The procedure continues until the input and output values for $\left\langle\rho_{f}^{Q}\right\rangle$ converge. As a consequence of symmetry the expectation value $\left\langle\rho_{f}^{Q}\right\rangle$ is independent of the sublattice $\left\{n_{p}\right\}$ chosen for calculations, but the Hamiltonian and eigenvectors do depend on the choice. This is because the quadrupoles have different orientations for different sublattices.

For nonzero temperature $T$, the mean-field equation for $\left\langle\rho_{f}^{Q}\right\rangle$ becomes

$$
\left\langle\rho_{f}^{Q}\right\rangle=\frac{1}{Z} \sum_{K} \rho_{f}^{Q}(K) e^{-\epsilon_{K} / T},
$$

where

$$
Z=\sum_{K} e^{-\epsilon_{K} / T}
$$

The results of the calculations are quoted in Tables VII and VIII, and in Figs. 2-4. We started by assuming $\lambda^{f f}$ $=-1750.5 \mathrm{~K}$, Table VI. The value is typical for the direct quadrupole-quadrupole electrostatic interaction. [In fact, it corresponds to the direct quadrupolar attraction in the $P a \overline{3}$ structure, i.e., $\lambda^{f f}=\lambda^{f f}(\operatorname{Pn} \overline{3} m)$, Table VI.] With this value we have found that the transition temperature $T_{Q}$ is only 0.44 K. A typical dependence of $\left\langle\rho_{f}^{Q}\right\rangle$ is shown in Fig. 2. The phase transition is of first order, with a discontinuity of the order parameter amplitude $\left\langle\rho_{f}^{Q}\right\rangle=-0.0075$ at $T_{Q}$. Comparing 
TABLE VIII. Mean-field (trigonal) splittings at $T=0$ with an enhanced quadrupolar interaction constant $\lambda_{e f f} / \lambda^{f f}=10 . \quad T_{Q}$ $=24.6 \mathrm{~K}, \rho_{0}=-0.0563$.

\begin{tabular}{ccccc}
\hline \hline$\Gamma$ & Deg. & $\left(\epsilon_{i}-\epsilon_{1}\right)(\mathrm{K})$ & $\mathcal{M}_{z}\left(\mu_{B}\right)$ & $\rho_{f}^{Q}$ \\
\hline$E$ & 2 & 0 & \pm 1.9211 & -0.0563 \\
$E$ & 2 & 54.0 & \pm 1.1723 & -0.0014 \\
$E$ & 2 & 72.8 & \pm 1.3684 & -0.0004 \\
$E$ & 2 & 85.8 & \pm 0.6211 & 0.0249 \\
$E$ & 2 & 122.1 & \pm 1.4893 & 0.0256 \\
\hline \hline
\end{tabular}

the present calculation with that for cerium, ${ }^{2}$ we observe that the low value of $T_{Q}$ is due to a small quadrupolar susceptibility of the ground state quartet $\Gamma_{8}$, since the difference in $\lambda$ is not that much (for cerium $\lambda^{f f}=-2241 \mathrm{~K}$ ).

The calculated transition temperature is very small in comparison with the experimental value of $25 \mathrm{~K}$. We then conclude that the structural phase transition can be explained by including an indirect bilinear quadrupole coupling (superexchange interaction ${ }^{29}$ ). The microscopic consideration of the superexchange interaction is beyond the scope of the present work. Instead, we model it by increasing the value of $\lambda^{f f}$. In such a case $\lambda^{f f}$ becomes a phenomenological parameter which we denote as $\lambda_{e f f}$. By changing $\lambda_{\text {eff }}$ we change the transition temperature as shown in Fig. 3. We have found that the experimental value of $25 \mathrm{~K}$ is achieved for $\lambda_{e f f} / \lambda^{f f} \sim 10$, which indicates a substantial increase of the effective bilinear coupling, Eq. (3.24). The relevant parameters of such strong mean field are given in Table VIII.

Finally, we would like to mention that the mean-field calculations have been done assuming that the CEF is weak, i.e., $x_{e f f}=0$. Increasing $x_{\text {eff }}$ leads to an increase of the CEF splittings, which results in a strong suppression of the transition temperature $T_{Q}$, Fig. 4. Notice that at $x_{e f f}\left(\Gamma_{8} \rightarrow \Gamma_{6}\right)$ $=0.241$ the ground state changes to the $\Gamma_{6}$ doublet, Fig. 1, which is apparently unfavorable for the quadrupolar order. With further increase of $x_{e f f}$ beyond the $x_{e f f}\left(\Gamma_{8} \rightarrow \Gamma_{6}\right)$ point

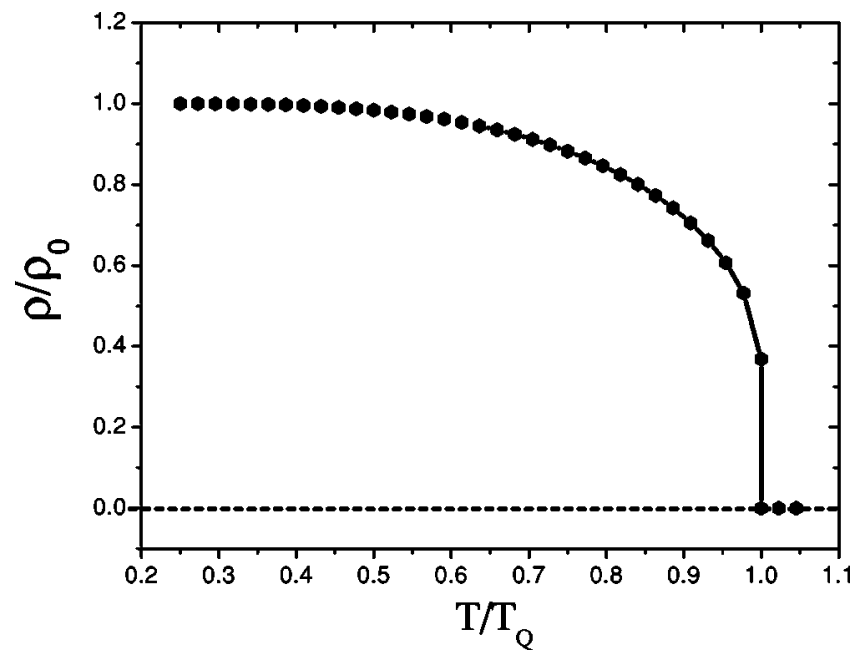

FIG. 2. A typical evolution of the order parameter amplitude $\left\langle\rho_{f}^{Q}\right\rangle$ with temperature; $\rho_{0}=\left.\left\langle\rho_{f}^{Q}\right\rangle\right|_{T=0}, T_{Q}$ is the transition temperature.

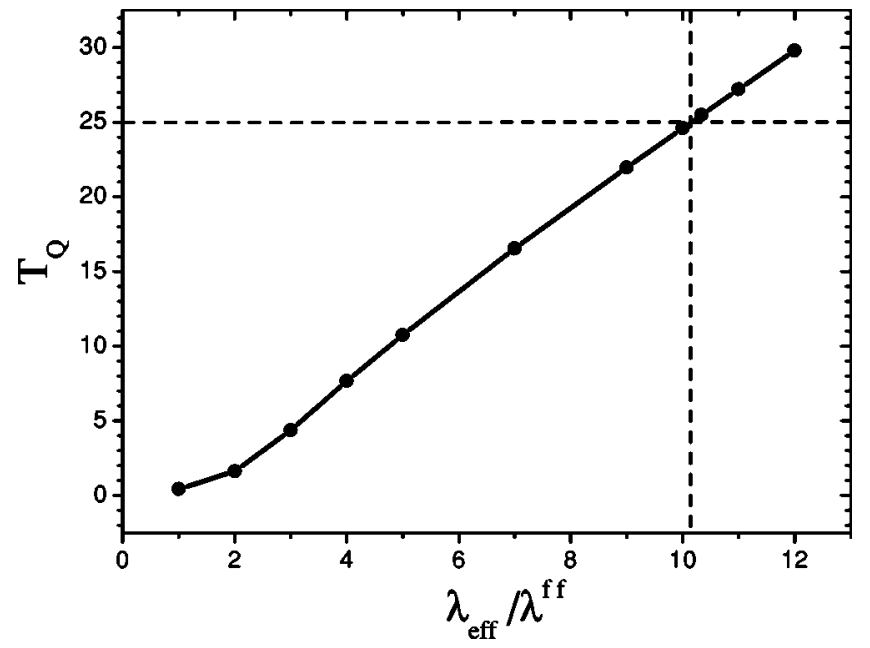

FIG. 3. The transition temperature $T_{Q}$ as a function of the quadrupolar interaction constant $\lambda_{\text {eff }}$.

the transition temperature goes quickly to zero and the ordered phase disappears.

\section{FOUR-ELECTRON CONFIGURATIONS}

As follows from the previous section, the model which takes into account only the $5 f^{3}$ configuration at each neptunium site is not able to explain the disappearance of the magnetic moments in the ordered phase at temperatures $T$ $<25 \mathrm{~K}$. On the other hand, the charge distribution inside the MT sphere centered at the neptunium nucleus indicates that there is always approximately one valence electron instantaneously present along with the three localized $5 f$ electrons, Table I. Even if the electron is in the $s$-orbital state, it experiences strong coupling with the $5 f$ electrons via intrasite $s$ $f$ transitions. Therefore, the excitation spectrum at each neptunium site differs from that for $5 f^{3}$ considered in Sec. III. In the following, we model the couplings with the valence electron by considering $7 s 5 f^{3}, 7 p 5 f^{3}$, and $6 d 5 f^{3}$ instantaneous configurations. Here, we will not study the mean field in

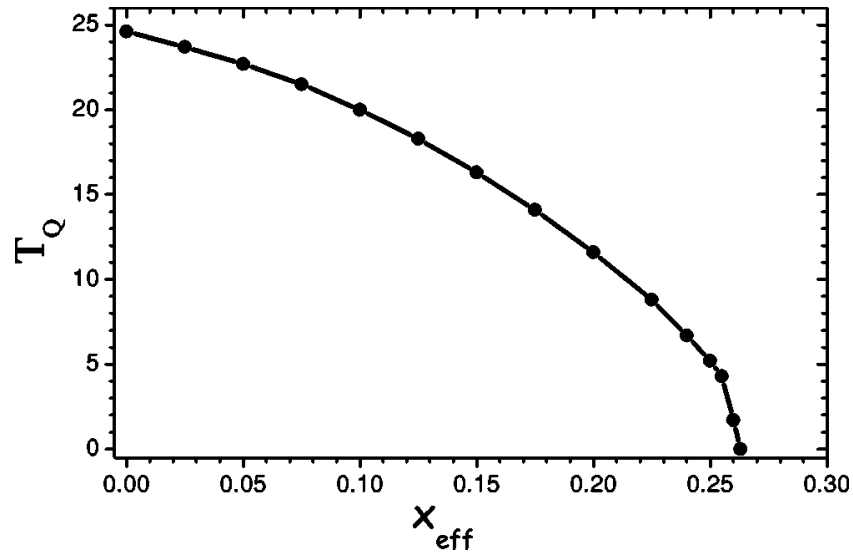

FIG. 4. The suppression of the transition temperature $T_{Q}$ with the increase of the CEF strength, $x_{e f f}$; see also Fig. 1 and Sec. III B; $\lambda_{e f f} / \lambda^{f f}=10$. 
TABLE IX. The five lowest and the highest eigenvalue of $7 s 5 f^{3}$; $g$ is the Landé factor.

\begin{tabular}{ccccc}
\hline \hline & Term & Deg. & $g\left(\mu_{B}\right)$ & $E$ \\
\hline 1 & ${ }^{5} I_{4}$ & 9 & 0.6266 & 0 \\
2 & ${ }^{5} I_{5}$ & 11 & 0.8983 & 196.5 \\
3 & ${ }^{5} I_{6}$ & 13 & 1.0670 & 737.2 \\
4 & ${ }^{5} F_{5}$ & 11 & 0.8700 & 814.4 \\
5 & ${ }^{5} I_{7}$ & 15 & 1.1691 & 1245.1 \\
$\ldots$ & $\ldots$ & $\ldots$ & $\ldots$ & $\ldots$ \\
82 & ${ }^{1} F_{0}$ & 7 & 1.0172 & 8229.5 \\
\hline \hline
\end{tabular}

such detail as for the $5 f^{3}$ configuration. Our main objective is to demonstrate that the ground state can be nonmagnetic and separated from the magnetic excitations by an energy gap larger than $25 \mathrm{~K}$. This means that at $T<25 \mathrm{~K}$, the magnetic moments are ineffective.

\section{A. $7 s 5 f^{3}$ configuration}

The $7 s 5 f^{3}$ configuration has been considered in detail in Sec. II. We are working here in the space of 728 state vectors $|I\rangle=\left|i_{1}^{f}, i_{2}^{f}, i_{3}^{f} ; i^{s}\right\rangle$. The $5 f$ states are coupled to two $s$ states through the $f$-s transitions accompanied by the multipole Coulomb interactions with $l=3$. The strength of this interaction was estimated from the LDA calculation of a $\mathrm{Np}$ atom, Eq. (2.18b)

$$
v_{3}^{f s-s f}=1.313 \mathrm{eV} .
$$

The electron energy spectrum of the $7 s 5 f^{3}$ configuration consists of 82 distinct levels; see Table IX. In the cubic crystal field the two lowest levels $\left({ }^{5} I_{4}\right.$ and $\left.{ }^{5} I_{5}\right)$ are split as quoted in Table $\mathrm{X}$. Notice that the ground state is nonmagnetic. However, at higher temperatures $(T>25 \mathrm{~K})$ two low- lying, excitations of $T_{2}$ and $T_{1}$ symmetry contribute to the Curie law for the magnetic susceptibility. ${ }^{30}$

In the ordered phase the local symmetry of the $\mathrm{Np}$ site changes to $D_{3 d} \cdot{ }^{13,28}$ As we discussed in Sec. III C the mean field can be expanded in a multipole series and, from sym-

TABLE X. CEF low energy spectrum and magnetic moments of the $7 s 5 f^{3}$ configuration of Np, $\Delta \epsilon=2293.2 \mathrm{~K}$. CEF parameters $B_{4}$ $=-288.1 \mathrm{~K}, B_{6}=254.2 \mathrm{~K} ; x_{e f f}=0$.

\begin{tabular}{ccccc}
\hline \hline & $\Gamma$ & Deg. & $\left(\epsilon_{i}-\epsilon_{1}\right)(\mathrm{K})$ & $\mathcal{M}_{z}\left(\mu_{B}\right)$ \\
\hline${ }^{5} I_{4}$ & $E$ & 2 & 0 & $0 ; 0$ \\
& $T_{2}$ & 3 & 15.6 & $\pm 1.5671 ; 0$ \\
& $T_{1}$ & 3 & 25.1 & $\pm 0.2946 ; 0$ \\
& $A$ & 1 & 61.6 & 0 \\
${ }^{5} I_{5}$ & $T_{1}$ & 3 & $\Delta \epsilon$ & $\pm 2.2435 ; 0$ \\
& $E$ & 2 & $\Delta \epsilon+4.1$ & $0 ; 0$ \\
& $T_{2}$ & 3 & $\Delta \epsilon+6.5$ & $\pm 2.2393 ; 0$ \\
& $T_{1}$ & 3 & $\Delta \epsilon+20.3$ & $\pm 2.7113 ; 0$ \\
\hline \hline
\end{tabular}

TABLE XI. Mean-field (trigonal) splittings of $7 s 5 f^{3}$ at $T=0$, $\Lambda^{f}=5252 \mathrm{~K}$

\begin{tabular}{cccc}
\hline \hline$\Gamma$ & Deg. & $\left(\epsilon_{i}-\epsilon_{1}\right)(\mathrm{K})$ & $\mathcal{M}_{z}\left(\mu_{B}\right)$ \\
\hline$A$ & 1 & 0 & 0 \\
$E$ & 2 & 26.0 & \pm 0.3467 \\
$E$ & 2 & 124.1 & \pm 0.6786 \\
$A$ & 1 & 301.8 & 0 \\
$A$ & 1 & 330.1 & 0 \\
$E$ & 2 & 516.5 & \pm 1.4769 \\
\hline \hline
\end{tabular}

metry, it follows that the strongest interaction is of quadrupolar type

$$
U^{Q Q}\left(\vec{n}_{p}\right)=-\Lambda^{f} \rho_{f}^{Q}\left(\vec{n}_{p}\right),
$$

where $\Lambda^{f}=\lambda^{f f}\left\langle\rho_{f}^{Q}\right\rangle$ [compare with (3.24)]. The quadrupolar operator $\rho_{f}^{Q}\left(\vec{n}_{p}\right)$ belonging to the sublattice $\left\{n_{p}\right\}$ is given again by Eq. (3.19) with the corresponding orientational function $\mathcal{S}_{p}$, Eqs. (3.17a)-(3.17d). The only difference is that the $s$ electron produces an additional Kronecker symbol, i.e., in Eq. (3.19) $\beta=1-3$. Notice that both CEF, Eq. (3.10a), and the mean field, Eq. (4.2), act only on the $5 f$ electrons. Taking $\Lambda^{f}=5252 \mathrm{~K}$, we diagonalized the full Hamiltonian

$$
H^{M F}(\vec{n})=U^{Q} Q_{(\vec{n})}+V_{C F}(\vec{n})+H_{\text {intra }}(\vec{n}) .
$$

The resultant electronic spectrum is shown in Table XI. We observe that the ground state is nonmagnetic, while the first magnetic excitation $(E)$ does not contribute to the magnetic susceptibility if $T<25 \mathrm{~K}$.

\section{B. $7 p 5 f^{3}$ configuration}

In the case of the $7 p 5 f^{3}$ configuration, we construct (14 $\times 13 \times 12 / 3$ ! $) \times 6=2184$ basis vectors

$$
|I\rangle=\left|i_{1}^{f}, i_{2}^{f}, i_{3}^{f} ; i^{p}\right\rangle .
$$

As before, $i^{f}$ refers to a $5 f$ electron, $i^{p}=\left(k, s_{z}\right)$ to the $7 p$ electron ( $k$ is its orbital part, $k=1-3$, and $s_{z}$ is the spin part).

The interaction between $5 f$ electrons was described in detail earlier. In addition, $p-p$ and $f-f$ transitions lead to intrasite multipole interactions with the $l=0$ and $l=2$ components; $f$ - $p$ transitions give the Coulomb multipole couplings with $l=2$ and $l=4$. The relevant Slater parameters were extracted from the radial dependences of a $\mathrm{Np}$ ion

$$
v_{2}^{f p-p f}=1.442, \quad v_{4}^{f p-p f}=0.739, \quad v_{2}^{f f-p p}=5.237 \quad(\text { in eV }) .
$$

Also, the $p$ electron experiences the spin-orbit interaction with $\zeta_{p}=1.2795 \mathrm{eV}$.

First, we calculated the electron spectrum of the free ion, $H_{\text {intra }}$ and obtained 242 distinct levels, Table XII. In the cubic crystal the ${ }^{5} K_{4}$ and ${ }^{5} I_{4}$ levels are split as quoted in Table XIII. (The procedure of CEF effects is outlined in Sec. III B; CEF does not act on the $p$ electron.) Notice that the ground state now is a magnetic triplet of the $T_{2}$ symmetry, which together 
TABLE XII. The five lowest and the highest eigenvalue of $7 p 5 f^{3} ; g$ is the Landé factor.

\begin{tabular}{ccccc}
\hline \hline & Term & Deg. & $g\left(\mu_{B}\right)$ & $E$ \\
\hline 1 & ${ }^{5} K_{5}$ & 11 & 0.7430 & 0 \\
2 & ${ }^{5} I_{4}$ & 9 & 0.7459 & 154.5 \\
3 & ${ }^{5} K_{6}$ & 13 & 0.9450 & 689.7 \\
4 & ${ }^{5} I_{5}$ & 11 & 0.9875 & 756.2 \\
5 & ${ }^{5} G_{2}$ & 5 & 0.5948 & 1248.1 \\
$\ldots$ & $\ldots$ & $\ldots$ & $\ldots$ & $\ldots$ \\
242 & ${ }^{3} P_{1}$ & 3 & 0.5031 & 10515.7 \\
\hline \hline
\end{tabular}

with two other magnetic $T_{1}$ excitations, Table XIII, gives the Curie law for the magnetic susceptibility.

At $T_{c}=25 \mathrm{~K}$ the structural phase transition occurs and the symmetry of the $\mathrm{Np}$ sites is reduced. ${ }^{13}$ This symmetry change is accompanied by lifting degeneracies of some cubic levels. ${ }^{28}$ In particular, the ground-state $T_{2}$ triplet is split into a doublet and a single level as demonstrated in Table XIV. The mean field is approximated by its quadrupolar electric part

$$
U^{Q} Q\left(\vec{n}_{i}\right)=-\Lambda^{f} \rho_{f}^{Q}\left(\vec{n}_{i}\right)-\Lambda^{p} \rho_{p}^{Q}\left(\vec{n}_{i}\right),
$$

where

$$
\begin{aligned}
& \Lambda^{f}=\lambda^{f f}\left\langle\rho_{f}^{Q}\right\rangle+\lambda^{f p}\left\langle\rho_{p}^{Q}\right\rangle, \\
& \Lambda^{p}=\lambda^{p f}\left\langle\rho_{f}^{Q}\right\rangle+\lambda^{p p}\left\langle\rho_{p}^{Q}\right\rangle .
\end{aligned}
$$

The following parameters of the interaction were assumed:

$$
\frac{q_{2}^{p}}{\left(R_{M T}^{\mathrm{Np}}\right)^{2}}=0.1604, \quad \Lambda^{f}=6612 \mathrm{~K}, \quad \Lambda^{p}=3426 \mathrm{~K} .
$$

Since the charge density expansion of the $p$ electron has quadrupolar components, $c_{k}\left(i^{p} j^{p}\right)=\left\langle i^{p}\left|\mathcal{S}_{k}\right| j^{p}\right\rangle \neq 0 \quad\left[\mathcal{S}_{k}\right.$ are given by Eqs. (3.17a)-(3.17d)], the mean-field expansion (4.6) includes the quadrupolar projection on $p$ states

TABLE XIII. CEF low energy spectrum and magnetic moments of the $7 p 5 f^{3}$ configuration of $\mathrm{Np}, \Delta \epsilon=1789.1 \mathrm{~K}$. CEF parameters $B_{4}=-288.1 \mathrm{~K}, B_{6}=254.2 \mathrm{~K} ; x_{e f f}=0$.

\begin{tabular}{ccccc}
\hline \hline & $\Gamma$ & Deg. & $\left(\epsilon_{i}-\epsilon_{1}\right)(\mathrm{K})$ & $\mathcal{M}_{z}\left(\mu_{B}\right)$ \\
\hline${ }^{5} K_{5}$ & $T_{2}$ & 3 & 0 & $\pm 1.8525 ; 0$ \\
& $T_{1}$ & 3 & 3.2 & $\pm 1.7788 ; 0$ \\
& $E$ & 2 & 16.7 & $0 ; 0$ \\
& $T_{1}$ & 3 & 45.0 & $\pm 2.1534 ; 0$ \\
${ }^{5} I_{4}$ & $E$ & 2 & $\Delta \epsilon$ & $0 ; 0$ \\
& $T_{2}$ & 3 & $\Delta \epsilon+14.8$ & $\pm 1.8645 ; 0$ \\
& $T_{1}$ & 3 & $\Delta \epsilon+24.8$ & $\pm 0.3655 ; 0$ \\
& $A$ & 1 & $\Delta \epsilon+59.1$ & 0 \\
\hline \hline
\end{tabular}

TABLE XIV. Mean-field (trigonal) splittings of $7 p 5 f^{3}$ at $T=0$; $\Lambda^{f}=6612 \mathrm{~K}, \Lambda^{p}=3426 \mathrm{~K}$.

\begin{tabular}{cccc}
\hline \hline$\Gamma$ & Deg. & $\left(\epsilon_{i}-\epsilon_{1}\right)(\mathrm{K})$ & $\mathcal{M}_{z}\left(\mu_{B}\right)$ \\
\hline$A$ & 1 & 0 & 0 \\
$E$ & 2 & 24.6 & \pm 0.4171 \\
$E$ & 2 & 105.8 & \pm 0.8134 \\
$A$ & 1 & 246.4 & 0 \\
$A$ & 1 & 272.6 & 0 \\
$E$ & 2 & 480.7 & \pm 1.7473 \\
\hline \hline
\end{tabular}

$$
\rho_{p}^{Q}\left(\vec{n}_{k}\right)=\sum_{I, J}|I\rangle \sum_{a} P(a) c_{k}\left(i^{p} j^{p}\right) \prod_{\beta=1}^{3} \delta_{i^{\prime} f^{j}{ }^{a}{ }_{\beta}}\langle J| .
$$

Here, $a$ is a permutation of $j_{1}^{f}, j_{2}^{f}, j_{3}^{f}$ to $j_{\beta}^{a f}, \beta=1-3 ; P(a)$ is the parity of the permutation. The quadrupolar operator for the $5 f$ electrons is given again by Eq. (3.19), where the index $\beta$ comprises the additional $p$ electron, i.e., $\beta=1-3$.

Notice that in the ordered phase the ground-state level is single and nonmagnetic, Table XIV. This mechanism can explain the loss of magnetic moments because the magnetic excitations of the $7 p 5 f^{3}$ configuration lie too high in energy.

\section{C. $6 d 5 f^{3}$ configuration}

The basis vectors here are

$$
|I\rangle=\left|i_{1}^{f}, i_{2}^{f}, i_{3}^{f} ; i^{d}\right\rangle
$$

where index $i^{f}$ stands for $5 f$ states $\left(i^{f}=1-14\right)$, while the index $i^{d}=\left(k, s_{z}\right)$ refers to five $d$ orbitals and the spin projection $s_{z}$. Thus, $i^{d}=1-10$, and in total there are 3640 nonequivalent basis vectors $|I\rangle$.

We start by considering the intrasite interactions $H_{\text {intra }}$. Here, in addition to $f$ - $f$ interactions, we distinguish two groups. The first group arises between $d$ - $d$ and $f$ - $f$ transitions. It is described by the multipole Coulomb repulsion with the $l=0,2$ and 4 angular components (SAFs). The second group is due to the $f$ - $d$ and $d$ - $f$ transitions. The corresponding multipole interactions are with $l=1,3$, and 5 . The relevant parameters were extracted from the LDA calculation of the $\mathrm{Np}$ ion in the $6 d 5 f^{3}$ configuration

$$
\begin{gathered}
v_{1}^{f d-d f}=11.322, \quad v_{3}^{f d-d f}=3.701, \quad v_{5}^{f d-d f}=1.794, \\
v_{2}^{f f-d d}=11.289, \quad v_{4}^{f f-d d}=3.482 \quad(\text { in } \mathrm{eV}) \\
\zeta_{d}=0.3497 \mathrm{eV} .
\end{gathered}
$$

The parameters for the $f$ - $f$ interactions were kept unchanged. We then diagonalized the $3640 \times 3640$ matrix of $\left\langle I\left|H_{\text {intra }}\right| J\right\rangle$ and obtained 383 distinct levels. The five lowest and the highest levels are quoted in Table XV. The CEF splittings of the lowest ${ }^{5} L_{6}$ and ${ }^{5} K_{5}$ levels are given in Table XVI. It should be noted that, unlike before, the CEF operator acts not only on the $5 f$ electrons but also on the $6 d$ one $^{2}$ 
TABLE XV. The five lowest and the highest eigenvalue of $6 d 5 f^{3} ; g$ is the Landé factor.

\begin{tabular}{ccccc}
\hline \hline & Term & Deg. & $g\left(\mu_{B}\right)$ & $E$ \\
\hline 1 & ${ }^{5} L_{6}$ & 13 & 0.7530 & 0 \\
2 & ${ }^{5} K_{5}$ & 11 & 0.7201 & 167.2 \\
3 & ${ }^{5} L_{7}$ & 15 & 0.9232 & 667.9 \\
4 & ${ }^{5} K_{6}$ & 13 & 0.9269 & 767.2 \\
5 & ${ }^{3} D_{3}$ & 7 & 0.6800 & 886.9 \\
$\ldots$ & $\ldots$ & $\ldots$ & $\ldots$ & $\ldots$ \\
383 & ${ }^{3} P_{1}$ & 3 & 1.0004 & 13116.4 \\
\hline \hline
\end{tabular}

$$
U^{C E F}(\vec{n})=B_{4}^{f} \rho_{f}^{4}(\vec{n})+B_{6}^{f} \rho_{f}^{6}(\vec{n})+B_{4}^{d} \rho_{d}^{4}(\vec{n})
$$

Here, $\rho_{f}^{l}(\vec{n}), l=4,6$, and $\rho_{d}^{4}(\vec{n})$ are cubic projectors on the $f$ and $d$ states, respectively, given by

$$
\begin{aligned}
& \rho_{f}^{l}(\vec{n})=\sum_{I, J}|I\rangle \sum_{a} P(a) \sum_{\alpha=1}^{3}\left\langle i_{\alpha}^{f}\left|K_{l}\right| j_{\alpha}^{a f}\right\rangle \prod_{\beta=1}^{3} \delta_{i_{\beta} j_{\beta}}\langle J|, \\
& \rho_{d}^{4}(\vec{n})=\sum_{I, J}|I\rangle \sum_{a} P(a)\left\langle i^{d}\left|K_{4}\right| j^{a d}\right\rangle \prod_{\beta=1}^{3} \delta_{i_{\beta}^{f}} j_{\beta}^{a f}\langle J|,
\end{aligned}
$$

where $K_{l}(\Omega)$ refers to the cubic harmonics with $l=4$ and 6 . Here, we keep the same notations as before [Eqs. (3.19) and (4.9)], i.e., the permutation $a$ transforms the indices $j_{1}^{f}, j_{2}^{f}, j_{3}^{f}$ to $j^{a f}{ }_{\kappa}, \kappa=1-3$. The permutations which interchange the $d$ and $f$ indices are excluded because they give zero contribution to $(4.13 \mathrm{a})$ and $(4.13 \mathrm{~b})$. The parameter $B_{4}^{d}$ was calculated by the method described in Sec. III B. For $x_{e f f}=0$, we have found that

TABLE XVI. CEF low energy spectrum and magnetic moments of the $6 d 5 f^{3}$ configuration of $\mathrm{Np}, \Delta \epsilon=1949.5 \mathrm{~K}$. CEF parameters $B_{4}=-288.1 \mathrm{~K}, B_{6}=254.2 \mathrm{~K}$, and $B_{4}^{d}=-232.7 \mathrm{~K} ; x_{e f f}=0$.

\begin{tabular}{ccccc}
\hline \hline & $\Gamma$ & Deg. & $\left(\epsilon_{i}-\epsilon_{1}\right)(\mathrm{K})$ & $\mathcal{M}_{z}\left(\mu_{B}\right)$ \\
\hline${ }^{5} L_{6}$ & $A$ & 1 & 0 & 0 \\
& $T_{2}$ & 3 & 9.7 & $\pm 0.1809 ; 0$ \\
& $A$ & 1 & 12.6 & 0 \\
& $T_{1}$ & 3 & 15.6 & $\pm 0.3772 ; 0$ \\
& $E$ & 2 & 36.4 & $0 ; 0$ \\
& $T_{2}$ & 3 & 43.6 & $\pm 2.0637 ; 0$ \\
& $T_{5}$ & 3 & $\Delta \epsilon$ & $\pm 1.7253 ; 0$ \\
& $T_{1}$ & 2 & $\Delta \epsilon+3.1$ & $0 ; 0$ \\
& $T_{2}$ & 3 & $\Delta \epsilon+21.1$ & $\pm 1.8006 ; 0$ \\
& $T_{1}$ & 3 & $\Delta \epsilon+25.3$ & $\pm 2.0883 ; 0$ \\
\hline \hline
\end{tabular}

TABLE XVII. Mean-field (trigonal) splittings of $6 d 5 f^{3}$ at $T=0$; $\Lambda^{f}=6220 \mathrm{~K}, \Lambda^{d}=3442 \mathrm{~K}$.

\begin{tabular}{cccc}
\hline \hline$\Gamma$ & Deg. & $\left(\epsilon_{i}-\epsilon_{1}\right)(\mathrm{K})$ & $\mathcal{M}_{z}\left(\mu_{B}\right)$ \\
\hline$A$ & 1 & 0 & 0 \\
$E$ & 2 & 32.8 & \pm 0.4362 \\
$E$ & 2 & 136.6 & \pm 0.8797 \\
$A$ & 1 & 311.2 & 0 \\
$A$ & 1 & 337.0 & 0 \\
$E$ & 2 & 601.2 & \pm 1.7673 \\
\hline \hline
\end{tabular}

$$
\frac{q_{4}^{d}}{\left(R_{M T}^{\mathrm{Np}}\right)^{4}}=0.1287, \quad B_{4}^{d}=-232.7 \mathrm{~K}
$$

Notice that for the ground state the CEF gives a nonmagnetic single level, Table XVI, but there are two low-lying magnetic levels $\left(T_{2}\right.$ and $\left.T_{1}\right)$ at 9.7 and $15.6 \mathrm{~K}$, which contribute to the Curie law of the magnetic susceptibility at $T>25 \mathrm{~K}$.

Below $25 \mathrm{~K}$ the local symmetry of $\mathrm{Np}$ is lowered. ${ }^{13}$ The mean field is given by

$$
U^{Q Q}\left(\vec{n}_{p}\right)=-\Lambda^{f} \rho_{f}^{Q}\left(\vec{n}_{p}\right)-\Lambda^{d} \rho_{d}^{Q}\left(\vec{n}_{p}\right)
$$

where

$$
\begin{gathered}
\Lambda^{f}=\lambda^{f f}\left\langle\rho_{f}^{Q}\right\rangle+\lambda^{f d}\left\langle\rho_{d}^{Q}\right\rangle, \\
\Lambda^{d}=\lambda^{d f}\left\langle\rho_{f}^{Q}\right\rangle+\lambda^{d d}\left\langle\rho_{d}^{Q}\right\rangle .
\end{gathered}
$$

Here again, $\rho_{f}^{Q}(\vec{n})$ and $\rho_{d}^{Q}(\vec{n})$ are quadrupolar projection on the $f$ and $d$ states, respectively. They are given by expressions similar to (4.13a) and (4.13b), where we replace $K_{l}\left(\hat{n}_{p}\right)$ by $\mathcal{S}_{p}$, Eqs. (3.17a)-(3.17d), for four sublattices $\left\{n_{p}\right\}=1-4$ of $P n \overline{3} m$, Sec. III C. [Compare also with (3.19) and (4.9).] Below we approximated the parameters of this interaction by

$$
\frac{q_{2}^{d}}{\left(R_{M T}^{\mathrm{Np}}\right)^{2}}=0.1713, \quad \Lambda^{f}=6220 \mathrm{~K}, \quad \Lambda^{d}=3442 \mathrm{~K} .
$$

We then diagonalized the whole Hamiltonian $H^{M F}\left[H^{M F}\right.$ $\left.=U^{Q} Q(\vec{n})+V_{C F}+H_{\text {intra }}\right]$ and obtained the lowest energy levels quoted in Table XVII. Notice that now the first magnetic excitation of $E$ symmetry is separated from the nonmagnetic ground state by an energy gap of $\sim 33 \mathrm{~K}$, which implies again disappearance of the Curie law for the magnetic susceptibility of the ordered phase $(T<25 \mathrm{~K})$.

\section{DISCUSSION AND CONCLUSIONS}

We have presented a multireference (many Slater determinant) approach to crystal-and mean field based on the technique of expanding the Coulomb repulsion between electrons in a multipolar series, Sec. II. The method is a genuine many-electron approach which requires a numerical classification of the permutations of the electrons on the same crystal site and a calculation of the Coulomb repulsion between all pairs. Thus, the fundamental group of electron 
permutations $^{31}$ is explicitly taken into account.

In the disordered phase we considered the Hamiltonian which includes the crystal electric field (CEF) effects and the intrasite Coulomb repulsion responsible for Hund's rules on equal footing, Sec. III. It is shown that the crystal field is reduced to a single particle potential. The crystal electric field levels above the ground state are in fact low-lying local excitations of the electron complex. The typical splittings there are of the order of $10 \mathrm{~K}$ and thus the electron spectrum is very sensitive to the crystal symmetry. An effective bilinear quadrupole-quadrupole Coulomb repulsion treated in the paper in the mean-field approximation competes with the crystal field and lowers the crystal symmetry at low temperature. The influence of both interactions (i.e., crystal field potential and the bilinear coupling) on the transition temperature has been investigated in detail for the $f^{3}$ configuration, Sec. III and Figs. 2-4.

Disappearance of magnetic moments in $\alpha$-Ce and $\mathrm{NpO}_{2}$ gives rise to a question of correlations between structural and magnetic properties in solids, which we have investigated theoretically in our model of the $\gamma-\alpha$ phase transition in Ce. ${ }^{1,2}$ The loss of magnetic moments in $\mathrm{NpO}_{2}$ at $25 \mathrm{~K}$ accompanied by a small volume contraction [0.018\% (Ref. 27)] has been known for many years, and was ascribed to an "isostructural" phase transition. Recently, however, it turned out that the phase change in $\mathrm{NpO}_{2}$ is a structural one, and the symmetry of the low temperature phase is $P n \overline{3} m$. In this respect it is interesting to notice that, in our model for $\mathrm{Ce}$, we have predicted the $P a \overline{3}$ space symmetry for the ordered $\alpha$ phase, ${ }^{1,2}$ which is very close to the $P n \overline{3} m$ structure reported for $\mathrm{NpO}_{2}$ in Ref. 13. In particular, the active irreducible representation also belongs to the $X$ point of the Brillouin zone.

In this paper we have pursued a simple idea that the symmetry lowering produces a splitting of the many-electron single site excitation spectrum, which can explain the difference in the behavior of the magnetic susceptibility and the loss of the magnetic moments. Before, such an idea was expressed by Friedt et al., Ref. 32. However, Friedt et al. applied it to the model of three $f$ electrons. In this case the three $f$ electrons cannot form a singlet state no matter what symmetry reduction occurs at low temperatures. The important element of the present study is that we have found a partial admixture of a conduction electron to the localized $f$ electrons. This fact is supported by our band structure calculation (Sec. II A) which indicates that, besides the three localized $5 f$ electrons, there is always approximately one conduction electron at each $\mathrm{Np}$ site. This changes the effective instantaneous configuration from the three-electron $5 f^{3}$ to a four-electron one $\left(7 s 5 f^{3}, 7 p 5 f^{3}\right.$, or $\left.6 d 5 f^{3}\right)$ and presents a possibility to obtain a nonmagnetic ground state without invoking the concept of the octupole order parameter. ${ }^{33}$ From this point of view it represents an alternative to the latter, and we believe that both approaches deserves a thorough experimental consideration and verification. A four-electron complex at the neptunium site can lead to a nonmagnetic ground state separated from the magnetic excitations by an energy gap $\sim 25 \mathrm{~K}$, Sec. IV. Perhaps the most clear example is the $7 p 5 f^{3}$ configuration (Tables XIII and XIV). In the disordered phase $(F m \overrightarrow{3} m$ space symmetry) the ground state is a triplet (Table XIII). The magnetic moments of the ground level are $\pm 1.8525 \mu_{B}$ and 0 . The first excited state located only at 3.2 $\mathrm{K}$ above the ground state is also magnetic $\left( \pm 1.7788 ; 0 \mu_{B}\right)$. In the ordered $(P n \overline{3} m)$ phase the ground state is a singlet (Table XIV) with zero magnetic moment. The first excited state is magnetic $\left( \pm 0.4171 \mu_{B}\right)$, but now it lies at $24.6 \mathrm{~K}$ above the ground level. That means that at temperatures below $\sim 25 \mathrm{~K}$ the first excited state is not populated and thus there are no effective magnetic moments at neptunium sites.

If we apply the present model to $\mathrm{UO}_{2}$, then at each uranium site one finds two localized $f$ electrons and approximately one conduction electron. In total, there will be approximately three electrons. It is not possible to construct a singlet state from three electrons. Therefore, the local moment will be omnipresent at low temperatures, which accounts for the magnetic behavior of $\mathrm{UO}_{2}$ in accordance with the experiment. ${ }^{34}$ This gives some credit to the presented model, although a nonmagnetic ground state of $\mathrm{PuO}_{2}$ (Ref. 19) requires additional consideration.

The general idea for the loss of magnetic moments to some extent is similar to the one suggested by models of Kondo and Anderson and often referred to as the Kondo effect. ${ }^{11}$ Notice, however, that here we are dealing with the intrasite interactions treated on the $a b$ initio level. In particular, we replace the Anderson hybridization, ${ }^{8}$ which is linear in terms of creation/annihilation operators for valence and localized electrons, by the Coulomb intrasite repulsion, which being a density-density coupling is bilinear in terms of these operators. Another important theoretical ingredient of our model is the symmetry lowering which modifies the excitation spectrum of the electron system at low temperatures. This part is absent in the Kondo mechanism. The presented picture of coupling between localized and delocalized electrons on the same site is close to the initial idea of Zener, who considered Hund's rules responsible for the coupling.9

CEF and mean field have been objects of theoretical interest for many years, ${ }^{21,22,26,35,36}$ and we would like to mention here some important relations between our model and other approaches. We have shown that CEF effects can be perceived as a first meaningful term of the intersite multipole expansion, when all neighbors of a neptunium site are considered in the spherical approximation $\left(l^{\prime}=0\right)$. It is then a single particle potential. ${ }^{21,22}$ The intersite nonspherical terms are also included in the full potential (FP) electron band structure calculations like FP-LMTO (linear muffin-tin orbital method) and FP-LAPW (linear augmented plane-wave method). ${ }^{4}$ Therefore, in principle one could say that the CEF effects are equivalent to the full potential treatment. ${ }^{23,24}$ However, there are two very important caveats here. First, in the band structure calculations the nonspherical terms of the potential apply to itinerant electrons in the ground state, while CEF effects are considered usually for localized electrons in the ground and excited states. The second and more important remark is that practically all band structure calculations are based on a single determinant approximation. This intrinsic feature does not allow one to describe the intrasite interactions fully. In particular, the atomic term structure and Hund's rules are excluded from the consideration. This shortcoming does not apply to our treatment, which is based on a many-determinant (multireference) approach. For the intrasite part of interactions our model is very close to the 
scheme described by Condon and Shortley for the electron spectra of atoms and ions, although there are some unimportant differences.

The main approximation of our approach is the choice of basis and electronic configuration. No additional approximations used sometimes for crystal field calculations are made. This distinguishes our approach from Stevens', ${ }^{26}$ where the CEF is expressed in terms of equivalent operators $J_{x}, J_{y}$, and $J_{z}$. The latter approach, as well as the work of Lea, Leask, and Wolf for cubic CEF (Ref. 25) based on it, are justified only if $J$ is a good quantum number. In our approach this condition is not necessary, and indeed a mixture of several $J$ values is allowed. Notice also that the approach of Stevens starts with the symmetry arguments, while the interactions are introduced later in a phenomenological manner.

However, the present calculation scheme does not take into account chemical bonding in an intrinsic way. Therefore, further development of the method should be focused on this problem.

\section{ACKNOWLEDGMENTS}

I am indebted to Professor K. H. Michel for valuable discussions on the problem. I also appreciate help from B. Ver- berck. This work has been financially supported by the Bijzonder Onderzoeksfonds, Universiteit Antwerpen (BOFNOI) and by the Fonds voor Wetenschappelijk Onderzoek, Vlaanderen.

\section{APPENDIX A: CORRECTION OF SLATER INTEGRALS FOR Np}

Since the experimental data on the energy splittings of the $4 f^{3}$ configuration of $\mathrm{Pr}^{3+}$ and $\mathrm{Nd}^{4+}$ are available from the atomic database of NIST, Ref. 10, while there is no such information for $5 f^{3}$, we have performed calculations of $v_{l}^{F-F}(l=2,4,6)$, Eq. (2.18a), by using the radial dependence of $\mathcal{R}_{f}$ obtained from LDA calculations of atoms. After this we diagonalized the Hamiltonian of the free ion $\left(V^{(3)}+H_{s o}\right)$ and compared our calculated spectra with the experimental ones. We have noticed that the comparison is improved (the sequence of terms corresponds to the experimental one) if we reduce $v_{2}^{F-F}$ and $v_{6}^{F-F}$ by a factor of 0.75 while keeping $v_{4}^{F-F}$ almost the same (factor of 0.975). Therefore, we have used the same scale factors for $\mathrm{Np}$ in $\mathrm{NpO}_{2}$ and obtained parameters given by Eq. (3.3).
*Also at: Institute of Physical Chemistry of RAS, Leninskii prospect 31, 117915 Moscow, Russia.

${ }^{1}$ A. V. Nikolaev and K. H. Michel, Eur. Phys. J. B 9, 619 (1999); 17, 363 (2000).

${ }^{2}$ A. V. Nikolaev and K. H. Michel, Phys. Rev. B 66, 054103 (2002).

${ }^{3}$ A. V. Nikolaev and K. H. Michel, J. Chem. Phys. 117, 4761 (2002).

${ }^{4}$ D. J. Singh, Planewaves, Pseudopotentials and the LAPW Method (Kluwer, Boston, 1994).

${ }^{5}$ E. U. Condon and G. H. Shortley, The Theory of Atomic Spectra (University Press, Cambridge, England, 1967).

${ }^{6}$ M. Wierzbowska, M. Lüders, and E. Tosatti, J. Phys. B 37, 2685 (2004).

${ }^{7}$ B. O. Roos and P. R. Taylor, Chem. Phys. 48, 157 (1980).

${ }^{8}$ P. W. Anderson, Phys. Rev. 124, 41 (1961).

${ }^{9}$ C. Zener, Phys. Rev. 81, 440 (1951); 82, 403 (1951).

${ }^{10}$ W. C. Martin, R. Zalubas, and L. Hagan, Atomic Energy LevelsThe Rare-Earth Elements, Natl. Stand. Ref. Data Ser., Natl. Bur. Stand. (U.S.) (U.S. GPO, Washington, D.C., 1978), Vol. 60, and original references therein; NIST Atomic Spectra Database (http://physics.nist.gov/cgi-bin/AtData/main_asd).

${ }^{11}$ P. Fulde, Electron Correlations in Molecules and Solids (Springer, Heidelberg, 1995).

${ }^{12}$ D. C. Koskenmaki and K. A. Gschneidner, Jr., Handbook on the Physics and Chemistry of Rare Earths, edited by K. A. Gschneidner, Jr. and L. Eyring (North-Holland, Amsterdam, 1978), p. 337.

${ }^{13}$ J. A. Paixão, C. Detlefs, M. J. Longfield, R. Caciuffo, P. Santini, N. Bernhoeft, J. Rabizant, and G. H. Lander, Phys. Rev. Lett. 89, 187202 (2002).

${ }^{14}$ G. V. Ionova and A. V. Nikolaev, Phys. Status Solidi B 162, 451
(1990).

${ }^{15}$ V. von Barth and L. Hedin, J. Phys. C 5, 1629 (1972).

${ }^{16}$ C. J. Bradley and A. P. Cracknell, The Mathematical Theory of Symmetry in Solids (Clarendon, Oxford, 1972).

${ }^{17}$ H. Yasuda and T. Yamamoto, Prog. Theor. Phys. 45, 1458 (1971); R. Heid, Phys. Rev. B 47, 15912 (1993).

${ }^{18}$ H. Eyring, T. L. J. Walter, and G. E. Kimball, Quantum Chemistry (Wiley, New York, 1944), pp. 240-241.

${ }^{19}$ P. Santini, R. Lémanski, and P. Erdös, Adv. Phys. 48, 537 (1999).

${ }^{20}$ G. Amoretti, A. Blaise, R. Caciuffo, D. Di Cola, J. M. Fournier, M. T. Hutchings, G. H. Lander, R. Osborn, A. Severing, and A. D. Taylor, J. Phys.: Condens. Matter 4, 3459 (1992).

${ }^{21}$ D. J. Newman, Adv. Phys. 20, 197 (1971), and references therein.

${ }^{22}$ D. J. Newman, J. Phys. F: Met. Phys. 13, 1511 (1983).

${ }^{23}$ M. Weinert, J. Math. Phys. 22, 2433 (1981).

${ }^{24}$ A. V. Nikolaev and P. N. Dyachkov, Int. J. Quantum Chem. 89, 57 (2002); 93, 375 (2003).

${ }^{25}$ K. R. Lea, M. J. M. Leask, and W. P. Wolf, J. Phys. Chem. Solids 23, 1381 (1962).

${ }^{26}$ K. W. H. Stevens, Proc. R. Soc. London 65, 209 (1952).

${ }^{27}$ D. Mannix, G. H. Lander, J. Rebizant, R. Caciuffo, N. Bernhoeft, E. Lidström, and C. Vettier, Phys. Rev. B 60, 15187 (1999).

${ }^{28}$ A. V. Nikolaev and K. H. Michel, Phys. Rev. B 68, 054112 (2003).

${ }^{29}$ P. W. Anderson, Phys. Rev. 79, 350 (1950); 115, 2 (1959).

${ }^{30}$ P. Erdös, G. Solt, Z. Zolnierek, A. Blaise, and J. M. Fournier, Physica B 102, 164 (1980).

${ }^{31}$ J. P. Elliott and P. G. Dawber, Symmetry in Physics (MacMillan, London, 1979), Vol. 2, Chap. 17.

${ }^{32}$ J. M. Friedt, F. J. Litterst, and J. Rebizant, Phys. Rev. B 32, 257 (1985).

${ }^{33}$ P. Santini and G. Amoretti, Phys. Rev. Lett. 85, 2188 (2000). 
${ }^{34}$ B. C. Frazer, G. Shirane, D. E. Cox, and C. E. Olsen, Phys. Rev. 140, A1448 (1965); B. T. M. Willis and R. I. Taylor, Phys. Lett. 17, 188 (1965).

${ }^{35}$ M. T. Hutchings, in Solid State Physics: Advances in Research and Applications, edited by F. Seitz and D. Turnbull (Academic, New York, 1964), Vol. 16, p. 227.

${ }^{36}$ J. Mulak and Z. Gajek, The Effective Crystal Field Potential (Elsevier, Amsterdam, 2000). 